\title{
The AcrB Efflux Pump: Conformational Cycling and Peristalsis Lead to Multidrug Resistance
}

\author{
Markus A. Seeger ${ }^{1,2,3}$, Kay Diederichs ${ }^{4}$, Thomas Eicher ${ }^{1}$, Lorenz Brandstätter ${ }^{1}$, André Schiefner ${ }^{4,5}$, \\ François Verrey ${ }^{1}$ and Klaas M. Pos ${ }^{1,6, *}$
}

\begin{abstract}
'Institute of Physiology and Zurich Centre for Integrative Human Physiology (ZIHP), University of Zurich, Winterthurerstrasse 190, CH-8057 Zürich, Switzerland; ${ }^{2}$ Institute of Microbiology, Swiss Federal Institute of Technology (ETH), Wolfgang-Pauli-Strasse 10, CH-8093 Zürich, Switzerland; ${ }^{3}$ Current address: Department of Pharmacology, University of Cambridge, Tennis Court Road, Cambridge CB2 IPD, United Kingdom; ${ }^{+}$Department of Biology, University of Konstanz, Universitätsstrasse 10, M647, D-78457 Konstanz, Germany; ${ }^{5}$ Present address: Department of Molecular Biology, BCC206, The Scripps Research Institute (TSRI), 10550 North Torrey Pines Road, La Jolla, CA 92037, USA and Current Address: Institute of Biochemistry and Cluster of Excellence Macromolecular Complexes, Johann Wolfang GoetheUniversity, Max-von-Laue-Str. 9, D-60438, Frankfurt am Main, Germany
\end{abstract}

\begin{abstract}
Antimicrobial resistance of human pathogenic bacteria is an emerging problem for global public health. This resistance is often associated with the overproduction of membrane transport proteins that are capable to pump chemotherapeutics, antibiotics, detergents, dyes and organic solvents out of the cell. In Gram-negative bacteria such as Escherichia coli and Pseudomonas aeruginosa, tripartite multidrug efflux systems extrude a large variety of cytotoxic substances from the cell membrane directly into the medium bypassing the periplasm and the outer membrane. In $E$. coli, the tripartite efflux system $\mathrm{Acr} A / \mathrm{AcrB} / \mathrm{TolC}$ is the pump in charge of the efflux of multiple antibiotics, dyes, bile salts and detergents. The trimeric outer membrane factor (OMF) TolC forms a $\beta$-barrel pore in the outer membrane and exhibits a long periplasmic $\alpha$-helical conduit. The periplasmic membrane fusion protein (MFP) AcrA serves as a linker between TolC and the trimeric resistance nodulation cell division (RND) pump AcrB, located in the inner membrane acting as a proton/drug antiporter.

The newly elucidated asymmetric structure of trimeric AcrB reveals three different monomer conformations representing consecutive states in a transport cycle. The monomers show tunnels with occlusions at different sites leading from the lateral side through the periplasmic porter (pore) domains towards the funnel of the trimer and TolC. The structural changes create a hydrophobic pocket in one monomer, which is not present in the other two monomers. Minocyclin and doxorubicin, both AcrB substrates, specifically bind to this pocket substantiating its role as drug binding pocket. The energy transduction from the proton motive force into drug efflux includes proton binding in (and release from) the transmembrane part. The conformational changes observed within a triad of essential, titratable residues (Asp407/Asp408/Lys940) residing in the hydrophobic transmembrane domain appear to be transduced by transmembrane helix 8 and associated with the conformational changes seen in the periplasmic domain.

From the asymmetric structure a possible peristaltic pump transport mechanism based on a functional rotation of the AcrB trimer has been postulated. The novel transport model merges Jardetzky's alternate access pump mechanism with the rotating site catalysis of $\mathrm{F}_{1} \mathrm{~F}_{\mathrm{o}}$ ATPase and suggests a working hypothesis for the transport mechanism of RND transporters in general.
\end{abstract}

\section{INTRODUCTION}

\section{Drug Efflux Transporters}

Based on a systematic genome-wide analysis of membrane transport systems, solute transporters were identified and classified into about 550 families and superfamilies. Five superfamilies comprising primary and secondary active drug transporters have been described and classified according to the approved transporter classification system [1]. These are the ATP binding cassette $(\mathrm{ABC})$ superfamily (TC\#3.A.1), the major facilitator superfamily (MFS) (TC\#2.A.1), the resistance/nodulation/division (RND) superfamily (TC\#2.A.6), the drug/metabolite transporter (DMT)

*Address correspondence to this author at the Institute of Physiology, University of Zurich, Winterthurerstrasse 190, CH-8057 Zürich, Switzerland Tel: +41-44-635 5046; Fax: +41-44-635 6814;

E-mail:kmpos@access.uzh.ch superfamily (TC\#2.A.7) and the multi antimicrobial extrusion (MATE) family (TC\#2.A.66) [2]. ABC drug transporters are primary active transporters and in eukaryotes they play a major role in extrusion of noxious substances out of the cell. In addition, transport of chemotherapeutics out of tumour cells is mediated by ABC-transporters (e.g. Pgp) and as a consequence causes failure of cancer therapy. LmrA of the Gram-positive bacterium Lactococcus lactis is a wellstudied prokaryotic $\mathrm{ABC}$ drug transporter, which transports different positively charged drugs [3]. Recently, a structure of its close homologue, Sav1866 of Staphylococcus aureus, was solved by X-ray crystallography at $3 \AA$ resolution [4]. The drug-translocating members of the other four superfamilies all belong to the class of electrochemical potentialdriven transporters (Fig. 1). Among the members of the MFS multidrug resistance transporters, MdfA is functionally well characterised and extrudes positively charged and neutral 


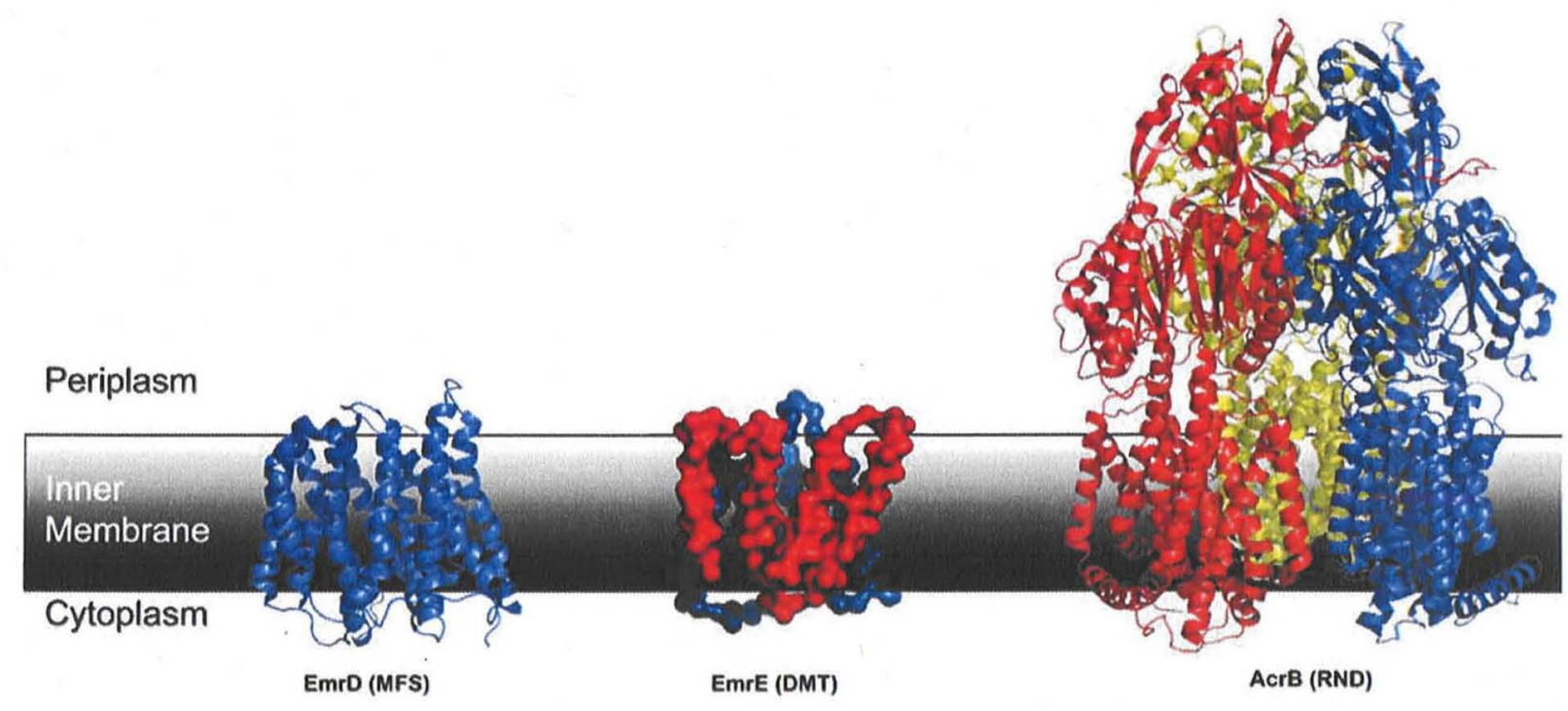

Fig. (1). Secondary active multidrug transporters. Structure models from members of the major facilitator superfamily (MFS), the drug/metabolite transporter (DMT) superfamily and the resistance nodulation division (RND) superfamily. From left to right: MFS: EmrD (PDB entry: 2GFP) DMT: EmrE (PDB entry: 3B62) and AcrB (PDB entries: 1IWG, 2GIF). Representative structures from 3 out of 4 secondary active multidrug transporter superfamilies are shown. There is no crystal structure available from the fourth family, the multi antimicrobial extrusion (MATE) family (e.g. NorM).

drugs [5]. The structure of its homologue EmrD has been recently solved at $3.5 \AA$ resolution [6]. Its structure appears similar to the structures of other MFS superfamily members such as LacY and GlpT [7, 8]. EmrE has been studied on the functional and structural level and belongs to the small multidrug resistance family (SMR) which is part of the drug/metabolite transporter (DMT) superfamily $[9,10]$. One member of the multi antimicrobial extrusion (MATE) family, NorM, is described as a sodium-ion driven efflux pump of Vibrio parahaemolyticus [11]. Drug transporting members of the RND superfamily, including AcrB, are among the most intensively studied transport systems.

\section{The RND Superfamily}

Members of the RND superfamily (TC\#2.A.6) are found ubiquitously in bacteria, archaea and eukaryotes. They mainly function as proton/substrate antiporters and belong to eight phylogenetic families [2]. Clustering patterns in these families of the RND superfamily correlate with substrate specificity. For example, the heavy metal efflux (HME) family (TC\#2.A.6.1) catalyzes export of heavy metal ions $\left(\mathrm{Zn}^{2+}\right.$, $\mathrm{Cu}^{2+}$ ) and the hydrophobe/amphiphile efflux-1 (HAE1) family (TC\#2.A.6.2) catalyzes the export of multiple drugs. The best characterized example of the HME family is CzcA from Ralstonia eutropha, acting as a heavy metal-ion/proton antiporter [12]. AcrB of Escherichia coli and MexB of Pseudomonas aeruginosa are the best studied members of the HAE1 family $[13,14]$. Human NPC1 being involved in lipid transport through the membrane and associated with the Niemann-Pick C1 disease belongs to the eukaryotic sterol transporter (EST) family (TC\#2.A.6.6) [15]. Most RND superfamily transporters consist of a single large polypeptide chain (700-1300 amino acid residues) and are predicted to span the membrane 12 times with two large periplasmic do- mains located between transmembrane helices 1 and 2 (TM1 and TM2) and between TM7 and TM8.

\section{RND/MFP/OMF Efflux Systems in Escherichia coli}

The RND members of the HME and HAE1 families function in conjunction with a membrane fusion protein (MFP; TC \#8.A.1) and an outer membrane factor (OMF; TC \#1.B.17) [2] (Fig. 2). The E. coli genome encodes 4 MFPs and 6 RND pumps. The genes acr $A B, \operatorname{acr} E F$ and $m d t E F$ coding for the MFP and RND members, respectively, are cotranscribed. On the other hand, the gene encoding the RND pump AcrD is found isolated on the chromosome. The $m d t A B C D$ operon consists of $m d t A$ encoding a MFP, $m d t B$ and $m d t C$ encoding two RND pumps and $m d t D$ encoding a MFS transporter. MFPs and RND proteins which are coded by co-transcribed genes form functional entities. However, some of the MFPs can form functional complexes with RND pumps in spite of the fact that the encoding genes are transcribed independently. Table 1 summarizes all known functional partnerships between the MFPs and RND pumps in $E$. coli. Remarkably, all pairs of MFPs and RND proteins in $E$. coli share $\mathrm{TolC}$ as outer membrane factor which is encoded independently elsewhere on the chromosome $[16,17]$. Systematic deletion of $E$. coli genes coding for RND, MFS, $\mathrm{SMR}$ and $\mathrm{ABC}$ transporter family members as well as MFPS and OMFs revealed that only the tripartite $\mathrm{Acr} A / \mathrm{AcrB} / \mathrm{TolC}$ efflux system contributes to the observed antibiotic resistance in $E$. coli wild type strains, whereas other transporters (including RND members) appear to play a minor role under standard laboratory conditions [18]. However, if the genes acr $D, \operatorname{acr} E F, m d t E F$ and $m d t A B C D$ encoding constituents of the AcrA/AcrD/TolC, AcrE/AcrF/TolC, MdtE/MdtF/TolC and $\mathrm{MdtA} / \mathrm{MdtB} / \mathrm{MdtC} / \mathrm{TolC}$ efflux systems, respectively, were overproduced in an $a c r B$ deficient background, partial 


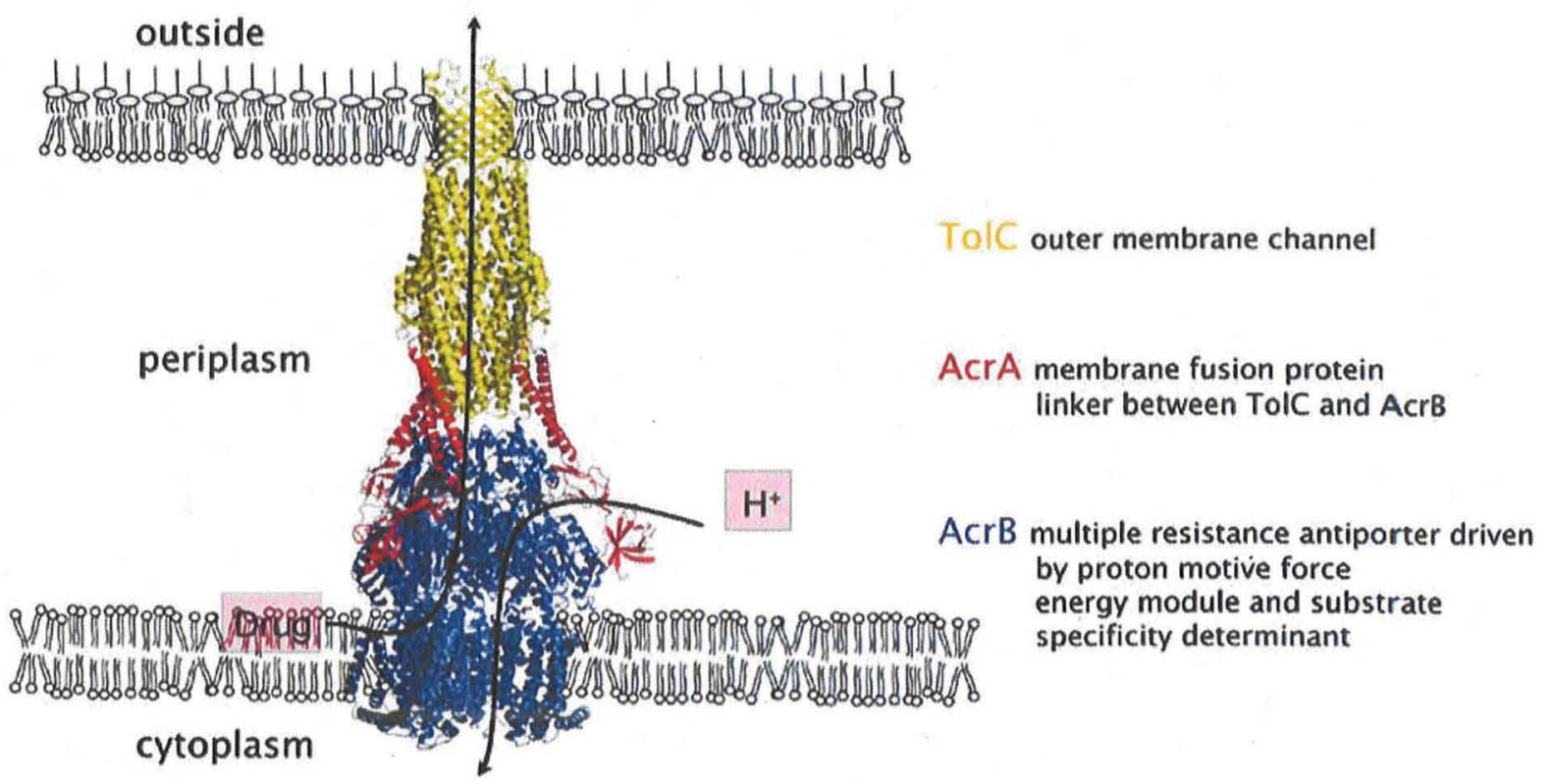

Fig. (2). Schematic drawing based on the X-ray structures of the tripartite multidrug efflux system AcrAB-TolC of Gram-negative E. coli. AcrB (RND component, in blue color) resides in the inner membrane and is responsible for substrate recognition/selection and energy transduction. Drugs are captured at the inner or outer leaflet of the inner membrane and extruded in a coupled exchange with protons. TolC (OMF component, yellow) forms a pore in the outer membrane which is extended by a long periplasmic conduit. AcrA (MFP component, red) mediates contact between $\mathrm{AcrB}$ and TolC. The presence of all three components is essential for the MDR phenotype.

recovery of the resistance for some of the tested drugs was observed [19]. It appears that the AcrA/AcrB/TolC system is constitutively expressed at a low level, whereas the other RND/MFP efflux systems are tightly repressed in E. coli. The acr locus coding for acr $A$ and $a c r B$ was mapped as early as in 1965 [20], when acriflavine sensitive E. coli strains were genetically analyzed. In addition, the mutants were shown to be susceptible to other dyes such as crystal violet and pyronine B. However, it took 28 years until Nikaido and co-workers postulated in 1993 that AcrA and AcrB function as components of an efflux pump system [21].

\section{RND/MFP/OMF Efflux Systems in Pseudomonas aerugi- nosa}

At around the same time as the research from the laboratory of H. Nikaido was published, Poole and co-workers [26] postulated that proteins encoded by genes of the $P$. aeruginosa operon mexAB-oprM constitute a multidrug efflux pump, where MexA is denoted the MFP, MexB the RND pump and OprM the OMF. Other than with TolC of E. coli, OprM is encoded by a gene located on the same operon as the other constituents of the $P$. aeruginosa tripartite efflux pump. Currently there are numerous tripartite RND/MFP/ OMF efflux systems characterized in $P$. aeruginosa named MexC/MexD/OprJ, MexE/MexF/OprN, MexX/MexY/OprM, MexJ/MexK/OprM, MexH/MexI/OpmD and MexV/MexW/ OprM. The coding genes of some of these tripartite systems are located on the same operon, whereas in others the corresponding OMF gene is located at a transcriptionally independent position on the chromosome (reviewed in [27]).

\section{Substrates of the Tripartite RND/MFP/OMF Efflux Sys-} tems

RND/MFP/OMF type efflux systems have been shown to exhibit a wide substrate specificity and extrude a broad range of chemically unrelated compounds including dyes, deter-

Table 1. Interacting Combinations of RND and MFP Components of E. coli. All RND/MFP Pairs Exclusively Recruit TolC as

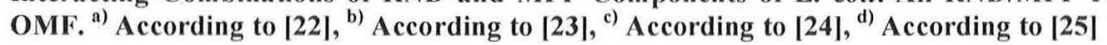

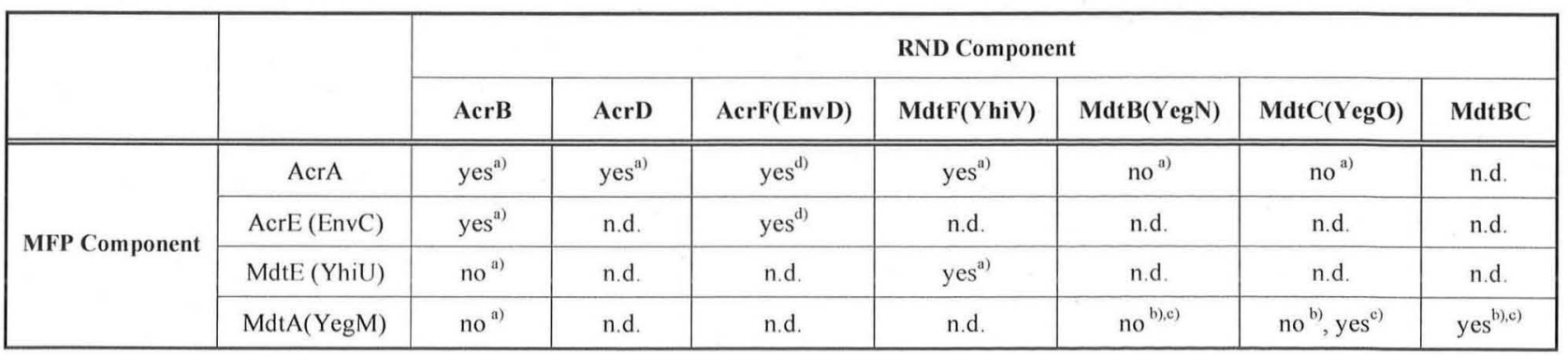


gents, bile salts, different classes of antibiotics and even organic solvents (Fig. 3) [19, 28-30]. The substrate molecules are structurally very diverse, can be charged or neutral and drastically differ in molecular weight. Some substrates are clearly amphiphilic i.e. having a large hydrophobic part and a small polar, often charged hydrophilic part (e.g. taurocholate, dodecylsulfate, oxacillin), whereas other substrates contain a delocalized charge within an aromatic environment (e.g ethidium, berberine, acriflavine). Yet others are simple aliphatic or alicyclic compounds (e.g. hexane, cyclohexane). The AcrA/AcrB/TolC efflux system transports antibiotics from a variety of classes including $\beta$-lactams (e.g. oxacillin), macrolides (e.g. erythromycin), fluoroquinolones (e.g. ciprofloxacin), oxazolidinones (e.g. linezolid) and tetracyclines (e.g. minocycline). The extent of AcrA/AcrB/TolC mediated activity on $\beta$-lactam resistance positively correlated with the lipophilicity of the side chain [29].<smiles>Cc1ccc2cc3cc(N)ccc3cc2c1</smiles>

Acriflavine<smiles>CCNC1C=C2OC3=CC(CC)C(C)CC3C(c3ccccc3OCC)C2C=C1C</smiles>

Rhodamine 6G

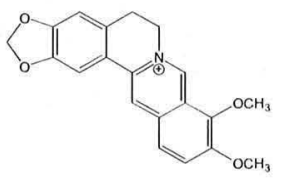

Berberine

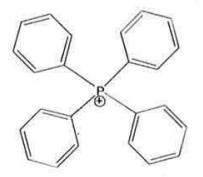

Tetraphenylphosphonium<smiles>CCOc1cc(C)ccc1-c1ccc(N)cc1C1CCCCC1</smiles>

Ethidium<smiles>O=C(O)C1=CN(C2CC2)C2CC(N3CCNCC3)C(F)CC2C1=O</smiles>

Ciprofloxacin

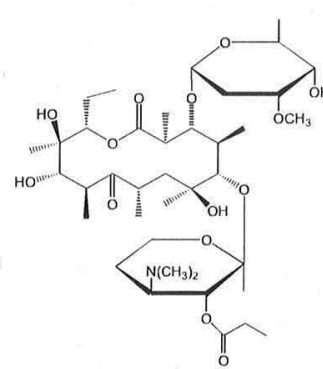

Erythromycin

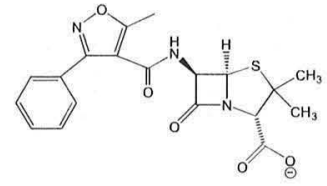

Oxacillin

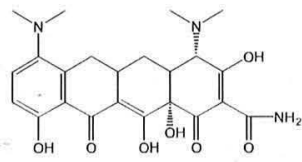

Minocycline
N-Phenylnaphthylamine (NPN)

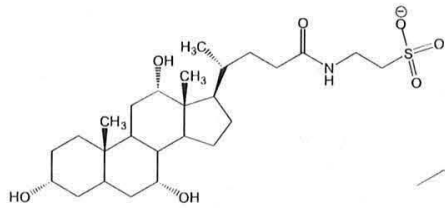

Taurocholate

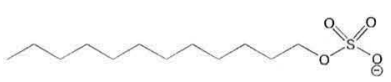

Dodecylsulfate
Hexane

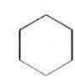

Cyclohexane

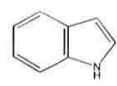

Indole

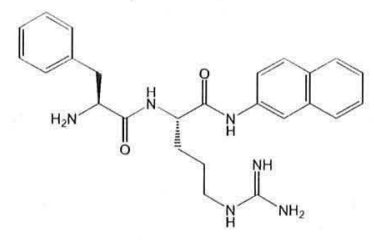

Phe-Arg- $\beta$-naphthylamide (PA $\beta N)$<smiles>c1ccc2c(CN3CCNCC3)cccc2c1</smiles>

1-(1-naphthylmethyl)-piperazine (NMP)

Fig. (3). Substrates and inhibitors of the AcrAB-TolC efflux system. The system confers resistance to a wide variety of noxious substances like dyes, different classes of antibiotics, detergents, bile salts and small organic molecules. Phe-Arg- $\beta$-naphthylamide and 1-(1naphthylmethyl)-piperazine (NMP) inhibit RND/MFP/OMF efflux systems. 
The question of the physiological role of these efflux systems in Gram-negative bacteria is still a matter of debate. It has been proposed that the efflux of bile salts allows the survival of Gram-negative bacteria in the gut [31], or that such efflux systems relieves the cell of toxic metabolites such as indole or from products of membrane and murein recycling $[32,33]$. More recently, the AcrA/AcrB/TolC efflux system was postulated to transport molecules mediating signals for quorum sensing [34]. An important question arises with respect to the broad substrate specificity and how the efflux system prevents transport of essential hydrophobic cofactors (e.g. flavins) or phospholipids out of the cell. Another line of investigation is the specific inhibition of these transporters by non-antibiotic compounds. Quite recently, inhibitors of the AcrA/AcrB/TolC and the MexA/MexB/ OprM efflux systems like Phe-Arg- $\beta$-naphthylamide or arylpiperazines have been postulated to inhibit the efflux of other substrates by high affinity competitive binding to the drug binding pocket $[35,36]$.

\section{Transport Across Two Membranes}

The contribution of RND/MFP/OMF efflux systems which span the two membranes of the Gram-negative cell to the intrinsic drug resistance is remarkable despite low expression of the pump constituents when compared to expression of drug pumps like the tetracycline transporter Tet, which appears to act as a single component in the inner membrane [37]. The ability of the RND/MFP/OMF efflux system to capture drugs from the inner membrane and the direct transport into the extracellular medium bypassing the periplasm results in a synergistic resistance effect [28]. In contrast, pumping activity of the inner-membrane transporter Tet would cause accumulation of tetracycline in the periplasm from where it easily diffuses over the cytoplasmic membrane back into the cell [31]. A recent report suggests that the tetracycline resistance phenotype by Tet is dependent on the presence of the AcrA/AcrB/TolC system [38]. The authors postulate Tet catalyzed transport of tetracycline into the periplasm, from where $\mathrm{Acr} A / \mathrm{AcrB} / \mathrm{TolC}$ pumps the antibiotic over the outer membrane.

\section{Clinical Relevance of Tripartite Efflux Systems}

RND/MFP/OMF efflux systems are associated with a major threat to human health as they play a central role in multiple antibiotic resistance of pathogenic Gram-negative bacteria such as Campylobacter jejuni, E. coli, Klebsiella pneumoniae, Acinetobacter baumannii, P. aeruginosa and Salmonella enterica serovar typhimurium (reviewed in [3941]). Highly resistant strains usually arise due to higher expression of the efflux pumps caused by mutations of regulatory proteins directly acting as repressors of transcription of the MFP/RND loci (e.g. AcrR) or indirectly by repressing the expression of upstream transcriptional activators (e.g. MarR repressing MarA) [42]. The presence of the efflux pump(s) facilitates the selection for high-level resistance through chromosomal mutations leading to target modification [40].

\section{Outer Membrane Factor (OMF) TolC}

The tolC (tolerance to $\underline{C}$ olicins) locus of $E$. coli was identified via a mutation causing a Colicin E1 resistant pheno- type. This mutant is also highly susceptible to bile salts and dyes [43]. TolC is postulated to act as Colicin E1 entry pore [44]. It also forms the outer membrane pore for HlyA toxin export by the type 1 secretion system in concert with an $\mathrm{ABC}$ transporter, HlyB, located in the inner membrane, and the membrane fusion protein HlyD [45]. Moreover, TolC acts as the OMF of all described RND/MFP/OMF efflux systems of E. coli (see above). Structures of several OMF members, TolC of E. coli, OprM of $P$. aeruginosa and VceC of Vibrio cholerae have been solved by X-ray crystallography at $2.1 \AA, 2.6 \AA$ and $1.8 \AA$ resolution, respectively [4648] (Fig. 4). TolC and its homologues form homotrimers presenting a $40 \AA$ OM located, pore forming $\beta$-barrel domain (inner diameters vary between $6-13 \AA$ in the different structures) and an $\alpha$-helical domain protruding $100 \AA$ into the periplasm, which is closed at the proximal end. Every monomer contributes 4 antiparallel $\beta$-sheets constituting the $\beta$-barrel domain of the trimeric structure and $4 \alpha$-helices, which pack in an left-twisted antiparallel arrangement to form the long periplasmic conduit [46]. The closed pore is known to open upon recruitment by substrate-laden complexes in the inner membrane during HlyA toxin export [45]. The transition to the open state of TolC is proposed to be achieved by an iris-like realignment of the helices at the tunnel entrance [49]. Mutants where intra- and intermolecular hydrogen bonds and salt bridges at the proximal end were abolished appeared to be completely functional with respect to HlyA export, but showed increased single channel conductance in black lipid bilayers [49]. Introduction of engineered disulfide linkages to avoid the opening of the proximal pore resulted in TolC mutants causing decreased drug and Hly A transport capacity [50]. In a screen selecting for TolC mutants causing decreased drug resistance, mutations resulted in a constitutively open TolC channel and were leading to influx of large antibiotics such as rifampin and vancomycin [51]. Further and new insights on TolC structure and function, described by B. Luisi and colleagues, can be found in this issue.

\section{Membrane Fusion Protein (MFP) AcrA}

The membrane fusion protein (MFP) family was given its name because of considerable sequence homology to the membrane fusion protein ( $\mathrm{F}$ protein) of paramyxovirus 5 [52]. Bacterial MFPs are essential partners for RND/MFP/ OMF efflux systems but also work in concert with transporters of the ATP binding cassette (ABC) superfamily [19, 45, 53 ] and members of the major facilitator superfamily (MFS) [28]. Two structures of bacterial MFPs, MexA of P. aeruginosa and AcrA of E. coli, have been solved by X-ray crystallography [47, 54, 55] (Figs. 4 and 7). In all three crystal structures (MexA was solved independently by two research groups) at resolutions between $2.3 \AA$ and $3 \AA$, only approximately two thirds of the amino acid residues of the MFPs could be assigned. A short $\mathrm{N}$-terminal tail and the $\mathrm{C}$ - terminal part of MexA (residues of unprocessed MexA 283-383 [54] or 302-383 [47], respectively) were highly disordered within the crystal or, in case of AcrA, had to be removed in order to obtain crystals. Sensitivity to proteolytic digest further supported the notion of the high flexibility of the Cterminal part $[55,56]$. The structures of the MexA and AcrA core proteins are divided into three parts, the $\beta$-barrel do- 


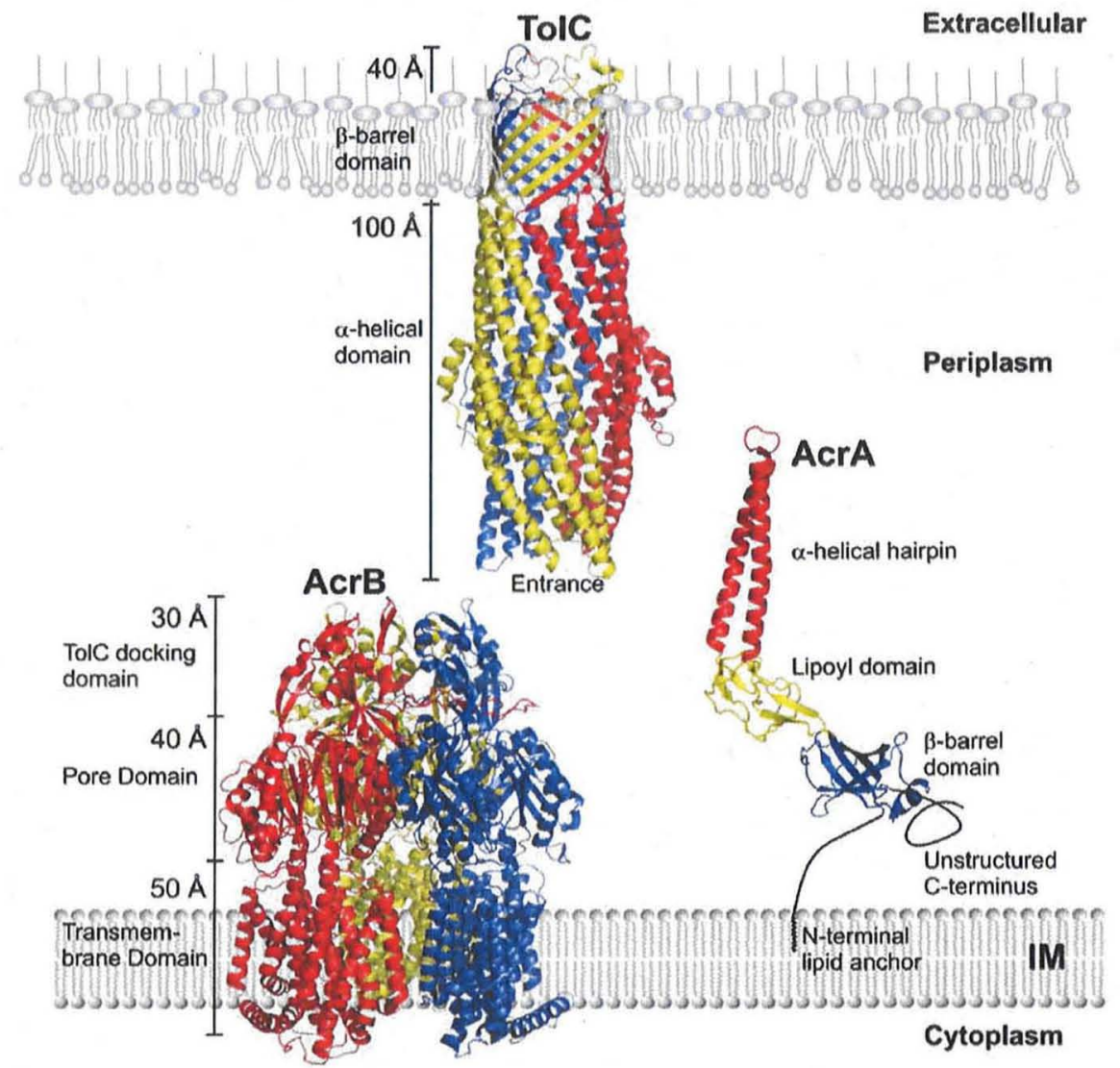

Fig. (4). Structures of the proteins constituting the tripartite AcrAB-TolC efflux machinery. AcrB (PDB entries: 1IWG, 2GIF) resides in the inner membrane (IM) and is composed of the transmembrane domain, the porter (pore) domain and the TolC docking domain. TolC (PDB entry: 1EK9) is integrated into the outer membrane (OM) with its $\beta$-barrel domain and forms a long conduit in the periplasm by its $\alpha$-helical domain which narrows to a closed entrance at the proximal end. $A \operatorname{crA}(2 \mathrm{~F} 1 \mathrm{M})$ is divided into the $\beta$-barrel domain, the lipoyl domain and the $\alpha$-helical hairpin. Its $\mathrm{N}$ - and $\mathrm{C}$-termini are not assigned due to missing electron density. AcrA is associated with the inner membrane via a $\mathrm{N}$ terminally attached lipid anchor Adapted from Eswaran et al. 2004.

main, the lipoyl domain and the $\alpha$-helical hairpin. Its $\mathrm{N}$ terminal signal sequence is responsible for the translocation of AcrA and MexA into the periplasm and is subsequently cleaved producing the mature protein. AcrA and MexA are attached to the inner membrane via a lipid anchor (palmitoylation) linked to a cysteine residue at the $\mathrm{N}$-terminus of the mature protein ( $\mathrm{C} 25$ and $\mathrm{C} 24$ of unprocessed AcrA and MexA, respectively). However, the membrane anchor appears not to be essential for drug efflux activity, as expression of soluble, non-membrane attached, periplasmically located mutants of the AcrA and MexA leads to a normal resistance phenotype $[57,58]$. AcrA was suggested to exist as a trimer, based on cross-linking data and blue-native gels, respectively $[59,60]$. MexA has been crystallized as a higher aggregate (tridecamer aggregate in the asymmetric unit) [47, 54] from purified samples containing mainly monomeric [47, 54] but also dimeric MexA (reported by Akama et al. [47]).

\section{Resistance Nodulation Division (RND) Pump AcrB}

RND proteins are the actual pumps of the tripartite RND/MFP/OMF efflux system, since both substrate specificity and energy transduction is attributed to this inner- membrane component. Although for many RND pumps the substrate specificity has been characterized, most of the structural and functional experiments were carried out on MexB of $P$. aeruginosa and AcrB of E. coli.

\section{Structure of AcrB}

The structure of trimeric AcrB has been initially solved at $3.5 \AA$ resolution in space group $\mathrm{R} 32$ with one monomer in the asymmetric unit [61-63] (Figs. 4 and 5). The AcrB monomer consists of 1049 amino acids and exhibits sequence homology and similar structural architecture between its $\mathrm{N}$ - and $\mathrm{C}$ - terminal half, indicating an early gene duplication event [52] (Fig. 6). Interestingly, co-expression of the individual $\mathrm{N}$ - and $\mathrm{C}$-terminal halves of the AcrB homologue MexB in trans (Residues 1-510 and 511-1049, AcrB numbering) resulted in an active RND-pump [64]. Full length AcrB contains twelve transmembrane $\alpha$-helices constituting the transmembrane domain (Fig. 5A, D). TM4 and TM10 are surrounded by the other transmembrane helices of the monomer and harbour the residues D407, D408 (TM4) and K940 (TM10) (Fig. 5D), which appear to play an essential role in proton translocation $[65,66]$. The connecting loops 

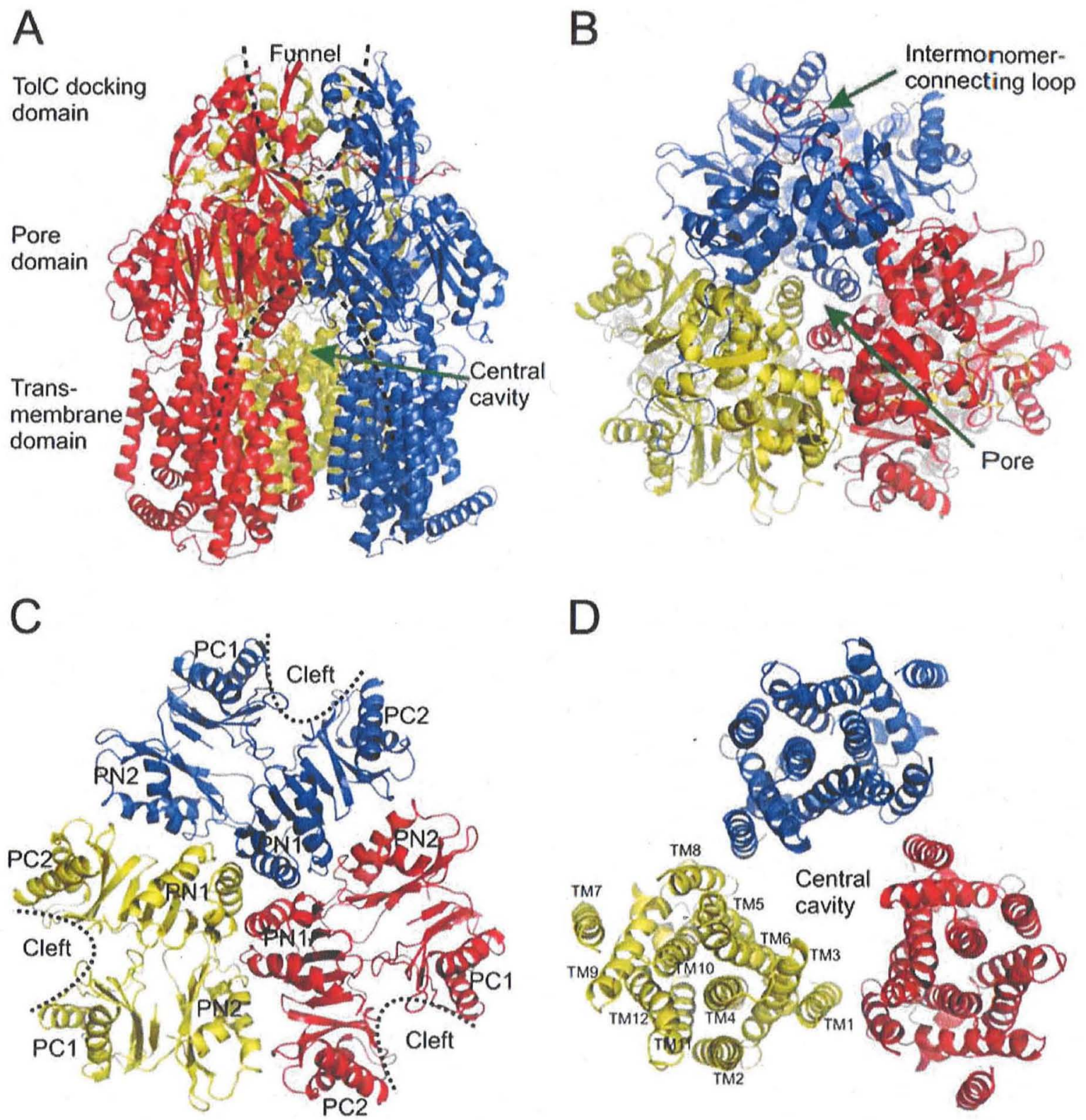

Fig. (5). A closer look to AcrB. (A) Side view. Trimeric AcrB is composed of the TolC docking domain exhibiting a funnel-like structure narrowing to a central pore located in the porter (pore) domain and the transmembrane domain confining a central cavity. (B) Top view. The trimeric state of AcrB is mainly stabilized by the intermonomer connecting loops. A central pore is formed by three $\alpha$-helices, donated by the PN1 subdomains of each AcrB monomer. (C) Top view onto the porter domain with its subdomains PN1, PN2, PC1 and PC2. The lateral cleft between subdomains PC1 and PC2 is suggested to accommodate AcrA, Murakami et al. 2002. (D) Top view onto the transmembrane domain. TM4 and TM10 are surrounded by other 10 transmembrane helices. The transmembrane helices of the monomers confine a large central cavity.

between TM1 and TM2 as well as TM7 and TM8 fold into two large periplasmic domains (Figs. $\mathbf{5 A}$ and $\mathbf{6}$ ), which account for approximately half of the amino acids of the full length AcrB molecule. The AcrB monomers form a trimer which appears to be stabilized by the intermonomer connecting loops protruding from the TolC docking domain into the adjacent monomer (Fig. 5B). The periplasmic part of AcrB consists of the TolC docking domain (divided into the DN and DC subdomains), which is located most distant from the membrane plane (Fig. 5A), and the porter (pore) domain. The TolC docking domain exhibits a funnel-like structure narrowing to a central pore located in the porter domain, the latter composed of subdomains PN1, PN2, PC1 and PC2 (Figs. 5A,C and 6). The characteristic central pore is formed by three $\alpha$-helices (designated pore helices), donated by the PN1 subdomains of each AcrB monomer (Fig. 5B,C). The pore has a small diameter and therefore does not allow drug passage in this conformation. The $\mathrm{PC1}$ and $\mathrm{PC} 2$ subdomains 


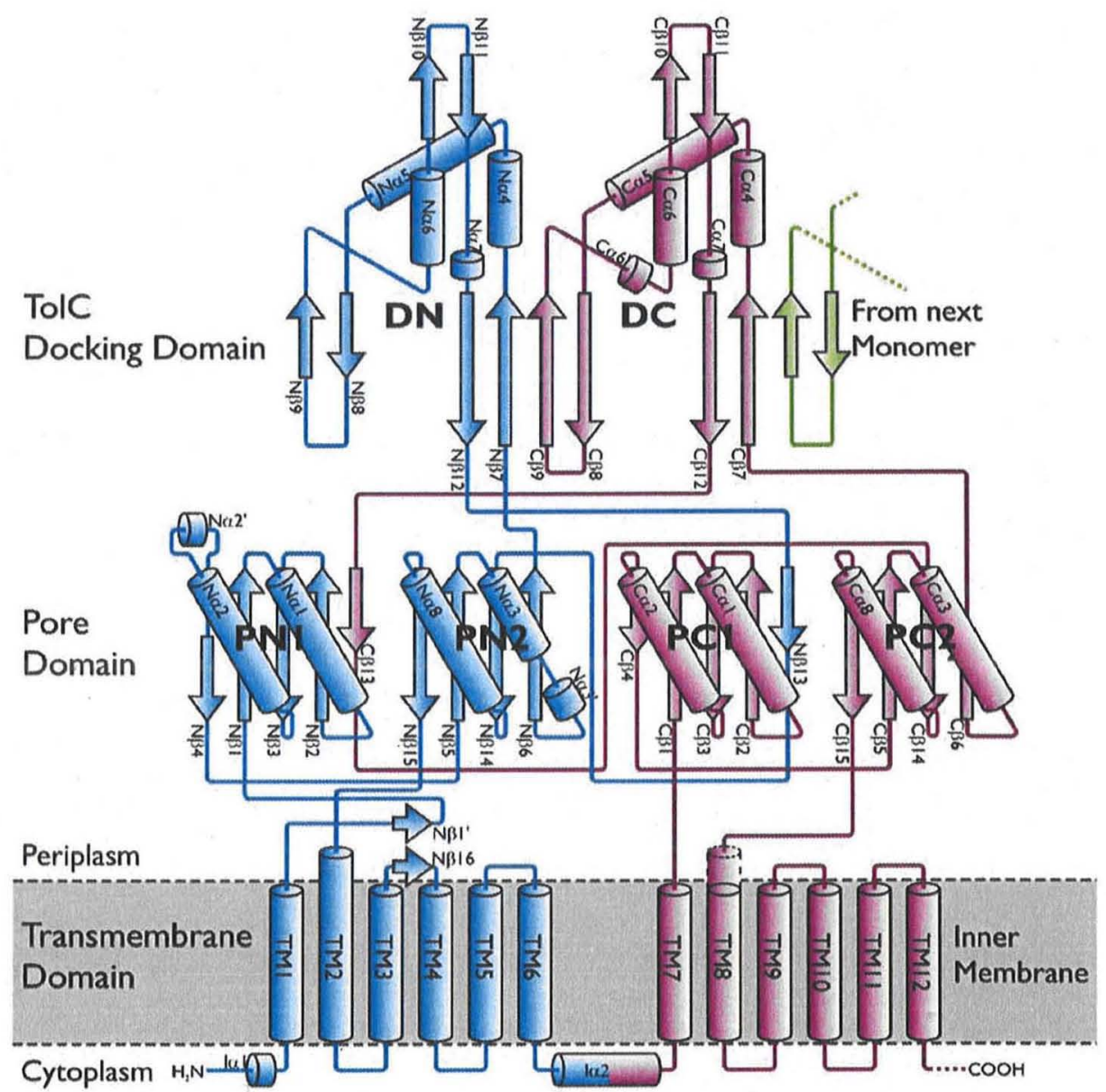

Fig. (6). AcrB monomer secondary structure scheme (residues 2-1033). The porter (pore) domain consists of four subdomains, PN1, PN2, PC1 and PC2. The TolC-docking domain has two subdomains, DN and DC. TM, transmembrane helices; N $\alpha, N \beta, C \alpha$ and $\mathrm{C} \beta$ are $\alpha$-helices and $\beta$-sheets of the $\mathrm{N}$-terminal part or the $\mathrm{C}$-terminal part of the periplasmic domain. I $\alpha 2$ is the cross- $\alpha$-helix at the cytoplasmic side. $\mathrm{N}$ - and $\mathrm{C}$-terminal halves are depicted in blue and magenta, respectively. The intermonomer connecting loop from the adjacent monomer is depicted in green. Adapted from Murakami et al., 2002 and extended with information drawn from the asymmetric AcrB structure (PDB entry: 2GIF).

constitute a cleft at the periplasmic periphery of the porter domain which is suggested to accommodate AcrA (Fig. 5C) [61]. At the interior of the protein near the membrane plane, the central pore leads to a central cavity and further to a 30 $35 \AA$ wide, presumably lipid-filled transmembrane hole defined by the ring-like arrangement of the TM helices of the trimer (Fig. 5A,D). Three vestibules at the monomer interface located just above the membrane plane lead towards the central cavity (Fig. 5A,D).

Recently, we [67] and others [68, 69] solved a structure of AcrB which does not entail three-fold symmetry of the trimer. In contrast to the symmetric structure, this asymmetric structure reveals three different monomer conformations representing consecutive states in a transport cycle, loose $(\mathrm{L})$, tight $(\mathrm{T})$ and open $(\mathrm{O})$ (see below). The structural changes in the T monomer create a hydrophobic pocket, which is not present in the other monomers. We [67] assumed that this pocket is a substrate binding pocket inside the porter domain and Murakami and colleagues [68] showed binding of minocyclin, 9-bromo-minocyclin and doxorubicin to this pocket (see below).

\section{Substrate Recognition}

The first symmetric structure of AcrB solved at $3.5 \AA$ was reported to be ligand free [61]. Another study from a different research group reported on four structures of symmetric AcrB-substrate complexes derived from diffraction data of crystals at 3.5-3.8 $\AA$ resolution [70]. Enforced by the three-fold symmetry of the crystal space group R32, three densities, which were interpreted as drug molecules binding to the AcrB trimer, were located in proximity to F386, F388, F458 and F459 in the central cavity. It was suggested that these structures represent the first binding step in the catalytic cycle before the drugs are further transported through the central pore towards TolC [70]. The interpretation of these results are, however, not beyond doubt due to the low resolution, the high $\mathrm{R}$ (and $\mathrm{R}_{\text {free }}$ )-factors and the sub-stoichiometric drug to AcrB molar ratio of 0.2 used in the cocrystallization assays. We described co-crystallization and crystal soaking experiments under very similar conditions of the same and other drugs at 10 to 100 times higher drug to AcrB ratios, but did not yield any indication of drug binding despite 2.8-2.9 $\AA$ resolution electron density map surveys 
[63]. Yet other crystal structures (obtained from crystals grown in R32 space group) by $\mathrm{Yu}$ et al. [71] showing ligand binding to a functionally active N109A mutant of AcrB. Drug binding was interpreted to occur at the lateral cleft near the hydrophobic residues F664 and F666. Data from the same crystals also included densities near the central cavity as has been reported earlier by the same group [70]. The derived structures were presented including 6 drug molecules bound to the AcrB trimer. Alanine substitution of F664 and F666 within the proposed periplasmic binding pocket led to substantial decrease in MIC values wheras on the other hand, functional characterization of mutants with alanine substituted F386, F388, F458 and F459 within the central cavity only showed marginal effects on efflux activity [71]. The latest published AcrB structure (derived from R32 crystals diffracting to $3.5 \AA$ ) by Törnroth-Horsefield et al. [72] includes a co-crystallized $\alpha$-helical peptide, which was identified as a fragment of YajC. This structure was published with six ampicillin molecules (originating from the selective growth medium) located inside the central cavity of the trimer despite the fact that the protein was purified and crystallized in the absence of this substrate.

Analysis of our R32 data at $2.7 \AA[63]$ showed that these crystals suffered from merohedral twinning [67]. A high twinning fraction results in blurred electron densities and hence makes interpretation of potentially bound substrates very difficult. Moreover, AcrB has been crystallized in the presence of dodecylmaltoside and cyclohexyl-hexylmaltoside, both known substrates of AcrB. The concentration of detergents inside the protein crystals is estimated to be approximately 20\% (DDM: $392 \mathrm{mM}$ ) and would present serious competition for other substrates present at low or sub $\mathrm{mM}$ concentrations. It should therefore be considered (especially with low resolution data) whether observed densities are derived from twinning artefacts and if not, whether the observed densities could represent sugar headgroups of the detergent attached to the protein. One exemplary approach to assign observed densities to bound substrates has been given by Murakami et al. [68]. This research group deployed 9bromo-minocyclin to make use of the anomalous signal produced by $\mathrm{Br}$ at $0.919 \AA$ and in this way elegantly showed the binding of 9-bromo-minocyclin to AcrB at relative low resolution (3.6 $\AA$ ). Binding of minocyclin, 9-bromo-minocyclin and doxorubicin to the asymmetric AcrB trimer occurred at a completely different site compared to the binding sites of the symmetric AcrB trimer at only one of the three monomers (T monomer) inside the porter domain. Strikingly, no substrate binding was observed in the central cavity of the asymmetric AcrB trimer.

Domain swapping experiments between RND-pumps with distinct substrate specificities (AcrB/AcrD, MexB/MexY, AcrB/MexB) already suggested that the substrate specificity is determined by the large periplasmic loops [73-75]. When both periplasmic loops of AcrD were exchanged with the corresponding amino acids of $\mathrm{AcrB}$, the chimeric protein transported the cognate substrates of $\mathrm{AcrB}$ but not those of AcrD. On the other hand, exchange of the TM2 to TM6 and TM8 to TM12 of AcrD with the corresponding residues of AcrB did not result in an AcrB transport phenotype [73]. The same result was obtained for the respective chimera of MexB and MexY. In addition, one by one exchange of TMl to
TM12 of MexB by the respective TMs of MexY always lead to chimeric transporters exhibiting substrate specificity of MexB [74]. Sequential replacement of the AcrB C-terminus by corresponding residues of $\mathrm{MexB}$ or the MexB N-terminus by the respective amino acids of AcrB suggested, that the Nterminal periplasmic loop between amino acids 60 to 612 is responsible for substrate specificity [75]. Six random mutants of MexD conferring increased resistance to carbenicillin, a poor substrate of wild type MexD, were mapped to both periplasmic loops [76]. These results are all in agreement with the key role of the periplasmic loops on substrate specificity.

\section{Elevator Mechanism}

Based on the symmetric AcrB structures derived from R32 crystals, it was hypothesized that diffusion of substrates occurs from the cytoplasmic site of the membrane via the transmembrane domain along a groove between TM8 and TM9 (for completely hydrophobic substrates except $\beta$ lactams) or from the periplasmic site of membrane via the vestibules (for $\beta$-lactams) into the central cavity. Subsequently, opening of the central pore would allow the transport of the substrates through the pore, to TolC and export to the external medium. Large conformational changes were postulated to be associated with this transport function, which was tentatively termed "elevator mechanism" [13]. Systematic cysteine scanning of the pore helix highlighted the importance of the pore helix side chains protruding into the central pore. The activity of AcrB pore mutants was substantially decreased due to intermolecular disulfide bridge formation between AcrB monomers [77]. However, a satisfactory interpretation of the latter results is also obtained in the context of the new structural $[67,68]$ and functional data [78], as will be discussed below.

\section{Energy Transduction}

Members of the RND superfamily are proposed to function as proton/substrate antiporters [2]. One of the most convincing experiment supporting this idea was carried out with reconstituted heavy metal-ion transporter $\mathrm{CzcA}$, a member of the heavy metal efflux (HME) family (TC\#2.A.6.1). Besides an observed fast facilitated diffusion of $\mathrm{Zn}^{2+}$ ions, a much slower proton $/ \mathrm{Zn}^{2+}$ antiport could be measured, which disappeared upon the mutation of D407 or E414 (AcrB numbering) [12]. These residues are presumed to play a central role in proton translocation in members of the HAE1 family (such as AcrB and MexB) as well $[65,66]$. Reconstituted AcrB in proteoliposomes has been shown to transport fluorescent phospholipids from donor to acceptor vesicles in the presence of a $\mathrm{pH}$ gradient. Addition of unlipidated, soluble AcrA facilitated this transport. Dissipation of the artificially generated proton gradient across the AcrB containing liposome membrane was accelerated in presence of substrates, indicating AcrB-mediated $\mathrm{H}^{+} /$drug antiport [79]. Reconstituted AcrD, a close homologue of AcrB, which transports rather hydrophilic aminoglycosides such as gentamycin, was able to translocate gentamycin in exchange for protons in presence of AcrA [80]. Systematic mutational analysis of all transmembrane domain localized, titratable residues of AcrB and MexB revealed that only five residues, D407, D408, K940, R971 and T978 (AcrB numbering) were essen- 
tial for drug transport $[65,66]$. These residues were suggested to constitute a charge network playing a central role in proton transduction, a notion supported by the AcrB structure [61, 67-69].

\section{The Tripartite RND/MFP/OMF Efflux System Forms a Multiprotein Complex}

The elimination of one gene of constitutively expressed tripartite RND/MFP/OMF efflux systems leads to a drug sensitive phenotype in Gram-negative bacteria. A growing number of publications deal with the mutual interaction of the single components within the complex.

\section{Biochemical Evidence}

Physical interaction between AcrA and AcrB [56, 59, 81], AcrA and TolC [51, 56, 81], AcrB and TolC [56, 81] and MexA and MexB [82] could be demonstrated using the cleavable cross-linker dithiobis(succinimidylpropionate) (DSP). Cross-linking efficiency between AcrA and AcrB was not dependent on the presence of TolC $[56,59,81]$, nor were cross-links between AcrA and TolC depending on the presence of AcrB [51, 56]. Moreover, interaction between AcrB and TolC was also detected in absence of AcrA [56, 83]. Using hetero-bifunctional cross-linkers, TolC containing single cysteine mutations on the lower $\alpha$-helical domain close to the entrance aperture could be cross-linked with AcrA. Reciprocally, site-specific cross-linking of AcrA cysteine variants to wild-type TolC identified the N-terminal $\alpha$ helix of the $\alpha$-helical hairpin of AcrA to be involved in the interaction with TolC [84]. Direct interaction between AcrB and TolC was demonstrated using disulfide cross-linking of cysteines located on distal loops of the TolC docking domain of AcrB with cysteines introduced at the proximal part of TolC [83]. The mutual interaction of the components of the tripartite complex does not depend on the presence of added substrates $[51,81,83]$, or the proton-motive force $(\mathrm{pmf})[56$, 81]. Moreover, the formation of the tripartite complex is not affected by mutations within proton translocation site of $\mathrm{AcrB}$, which render the protein pump inactive $[81,83]$. The tripartite MexA/MexB/OprM complex was co-purified from overproducing $E$. coli cells without the use of cross-linking agents by cobalt-chelate affinity chromatography. The overproduction of all three components of the tripartite efflux machinery was crucial to achieve co-purification of the complex [85]. Physical interaction between AcrA and TolC and between AcrA and AcrB was shown in vitro by isothermal titration calorimetry, whereas AcrB and TolC do not show interaction in this particular assay. Because of rather complex titration patterns non-linear regression of the data yielded two and four different dissociation constants for the interaction between AcrA and AcrB and between AcrA and TolC, respectively. The multiple $\mathrm{K}_{\mathrm{d}}$ values are proposed to represent sequential phases of the respective protein-protein interactions and range between $0.35 \mu \mathrm{M}$ and $17.2 \mu \mathrm{M}$ [56].

\section{Genetic Studies}

Genetic studies were in further support of the biophysical data described above and in addition were successfully exploited to map potential interacting domains between the components of the efflux system. MdtE (formerly YhiU) is an MFP of $E$. coli which operates in conjugation with TolC and MdtF, but not with AcrB (see Table 1). Chimeric analysis between both MFPs AcrA and MdtE revealed that a region within the C-terminal part of AcrA (residues 290-357, numbering of unprocessed AcrA throughout) interacts with AcrB [22]. In the AcrA crystal structure, most of this part (residues 300-357) is not resolved due to disorder [55]. A screen for rescue mutations of an inactive TolC mutant (P246R, S350C) within the acrRAB regulon y ielded colonies with mutations in AcrA (10 cases) and AcrR (one case), but not in AcrB [86]. The TolC P246R_S350C double mutant was shown to be partially defective in assembly, but in the presence of some of the selected AcrA mutants, its proper integration into the outer membrane was improved, i.e. it was stabilized in presence of mutant AcrA presumably due to a direct physical interaction. Interestingly, this assembly improvement was fully dependent on AcrB, because it could not be reproduced in an $a c r B$ negative background. All but one AcrA mutation map on the $\beta$-barrel domain [86] (Fig. 7). Mutations in MexA compromising antibiotic efflux of the MexA/MexB/OprM system mapped in the $\beta$-barrel domain of MexA as well, but were demonstrated to lead to an interaction loss with MexB. However, the effect of the mutations on OprM interaction/assembly was not tested [82] (Fig. 7). Screening for MexA mutants interplaying with OMF member OprN, which is not a cognate partner of MexA, revealed a mutation in the $\alpha$-helical hairpin of MexA suggesting that the latter domain is involved in interactions with the OMF (Fig. 7). This suggestion is even more appreciated since substitution of the $\alpha$-helical hairpin of MexA with the corresponding residues of MexE (which is the cognate partner of OprN) results in a functional heterologous MexA/MexB/ OprN efflux system [87]. Similarly, the substitution of the AcrA hairpin subdomain with that of MexA is sufficient to change the heterologous AcrA/AcrB/OprM hybrid efflux system from a non-functional into a functional export machine [88]. In a directed evolution approach, mutant TolC variants carrying single or double amino acid substitution were selected to form an active heterologous MexA/MexB/ TolC efflux pump. All substitutions leading to an active pump map to the periplasmic equatorial domain and entrance coiled coils of TolC and converge to the amino acid sequence of the native MexA/MexB partner OprM [89] (Fig. 7). Gain-of-function mutations within $\mathrm{VceC}$ resulted in functional heterologous AcrA/AcrB/VceC export machinery and mutations map as well at the entrance coiled coils of VceC. However, in this case no convergence towards the amino acid sequence of TolC could be observed [90]. In summary, the current data support the motion for a physical interaction of the MFP's $\alpha$-helical hairpin with OMF's coiled coils below the equatorial domain at the periplasmic tip.

In contrast, interactions between RNDs and MFPs are still poorly understood. MexB mutations resulting in an antibiotic-sensitive phenotype mapped at the lateral cleft of the periplasmic domain of MexB. Several mutations on the $\beta$ barrel domain of MexA suppress the MexB mutants, restore their pumping activity and confer resistance towards their host [91]. Very recent work [92] on the compatibility between MexB of $P$. aeruginosa and AcrA/TolC of E. coli surprisingly showed functional complementation for dodecylsulfate, novobiocin and ethidium, but not for other MexB 

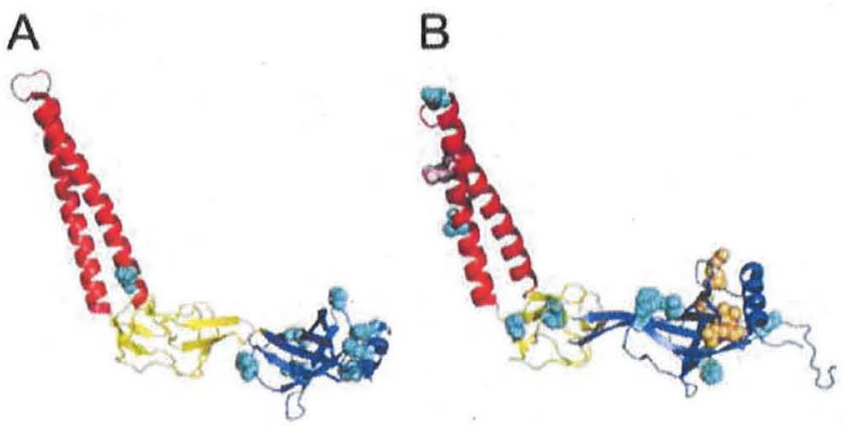

C

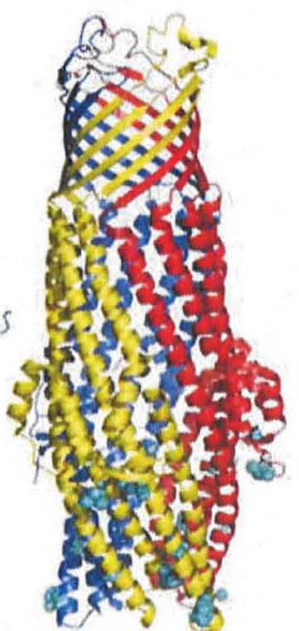

Fig. (7). Visualization of mutations in AcrA (A) and MexA (B) related to functional interactions with the RND and the OMF components of the tripartite efflux system. The $\beta$-barrel domain, lipoyl domain and the $\alpha$-helical hairpin are colored in blue, yellow and red, respectively. (A) Substitutions in AcrA that suppress a drug sensitive phenotype caused by mutant TolC (P246R_S350C) are shown as space fill representations and coloured in aquamarine (Gerken et al. 2004). (B) MexA residues which substitution caused a drug sensitive phenotype of MexAB-OprM, are shown as space fill representations and coloured in aquamarine (Nehme et al. 2004). Substitutions of residues involved in the rescue of a defective MexB mutant are coloured in light orange (Nehme et al. 2005). The substitutions of Q1 16R in MexA allowed functional interaction between MexA and the non-physiological OMF partner OprN and is indicated in a pink space filled representation (Eda $e t$ al. 2006). (C) Substitutions of residues in TolC that afforded an active MexAB-TolC efflux system are depicted as aquamarine space fill representations (Bokma et al. 2006).

substrates. Single substitutions in AcrA or a double mutation in MexB (T329I/A802V) were sufficient for improvement of the functional alignment between the two non-cognate subunits demonstrated by the markedly elevated MIC values of these mutants.

\section{In silico Modelling}

The availability of structures of all three individual components of the tripartite RND/MFP/OMF efflux system was exploited to predict the tripartite structure of the AcrA/AcrB/ TolC transport machinery. All models propose an assembly of trimeric AcrB and trimeric TolC co-axially along their three fold symmetrical axes (as in Fig. 2) but differ in the AcrA:AcrB:TolC stoichiometry. Because MexA was crystallized as a tridecameric ring-like structure, a ring of $9 \mathrm{MexA}$ monomers enclosing a MexB-OprM complex was suggested, i.e. a 3:1:1 stoichiometry [54]. Another model favours a 2:1:1 stoichiometry between MexA, MexB and OprM, with three MexA dimers oriented in a ring-like arrangement towards MexB-OprM [47]. Based on calculations and from disulfide cross-link studies, an analogous model was suggested for the AcrA/AcrB/TolC complex [88]. In support, the $2: 1: 1$ stoichiometry would account for the abundance of MexA relative to MexB and OprM in the $P$. aeruginosa cell, as quantified by immunoblotting [47]. In yet another in silico model, a 1:1:1 stoichiometry was suggested for the AcrA/ AcrB/TolC complex, which in contrast to the studies mentioned above permits direct contacts between AcrB and TolC [48]. The 1:1 stoichiometry between TolC and AcrA is supported by site-specific cross-linking data of single cysteine substituted TolC to AcrA and vice versa [84]. The experimental data allowed a data-driven docking approach to model the interaction surface between AcrA and TolC [84]. All models described above predict the interaction between the $\alpha$-helical hairpin of the MFP and the coiled coils of the $\alpha$ helical domain of the OMF and in addition, one study [48] predicts an interaction between the $\beta$-barrel of the MFP with the lateral cleft of the RND pump. A central role within the tripartite complex inheres to the MFP. Molecular dynamic simulation using the structure of MexA as a starting point revealed correlated movements between the $\beta$-barrel domain and the $\alpha$-helical hairpin, which are suggested to provide considerable flexibility within MexA, enabling it to dynamically bridge the RND and OMF [93]. The calculated movements would result in a hinge bending of about $19^{\circ}$ at the junction between the $\alpha$-helical hairpin and lipoyl domain (Figs. 4 and 7). Interestingly, comparison of the four AcrA monomer structures (PDB ID: $2 \mathrm{~F} 1 \mathrm{M}$ ) in the asymmetric unit of the AcrA crystal structure reveals a maximal difference of that hinge angle of about $15^{\circ}$. Despite all efforts, the exact stoichiometry and assembly of the tripartite RND/MFP/OMF efflux system remains elusive and crystal structures of the respective complexes have to be solved to get clearer evidence.

\section{DISCUSSION}

The Protein Data Bank (www.pdb.org) contains at the moment of writing $24 \mathrm{AcrB}$ structures. The first AcrB structure (year 2002, PDB entry: 1IWG) [61] was based on $3.5 \AA$ $\mathrm{X}$-ray diffraction data from R32 crystals containing one unliganded AcrB monomer in the asymmetric unit i.e. it describes a symmetric AcrB trimer. In the following years, other groups deposited symmetric (wildtype and mutant) AcrB X-ray structures (based on 3.1-3.8 $\AA$ data) with and 

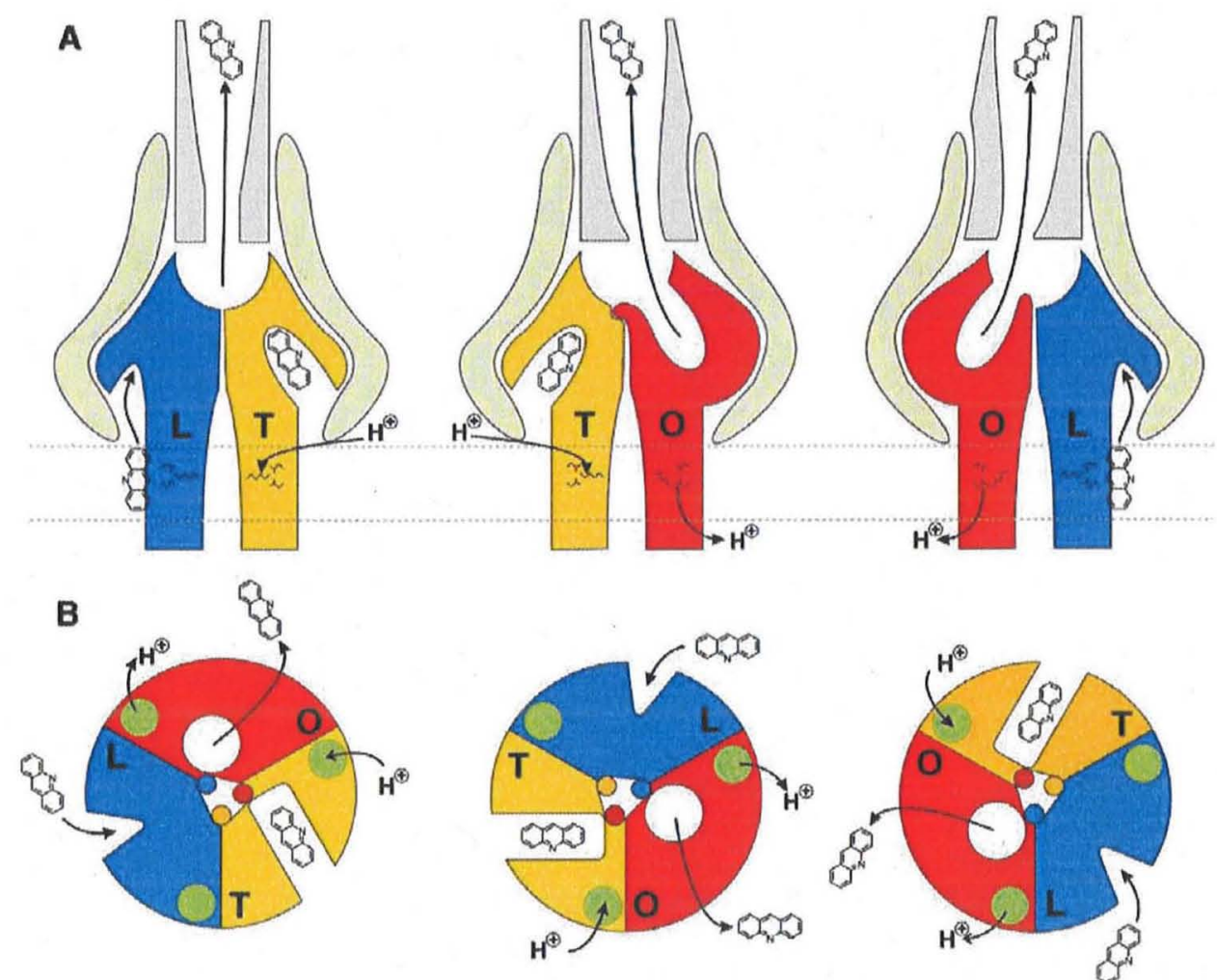

Fig. (8). Schematic representation of the AcrB alternating site functional rotation transport mechanism. The conformational states loose (L), tight $(\mathrm{T})$, and open $(\mathrm{O})$ are colored blue, yellow and red, respectively. (A) Side-view schematic representation of two of the three monomers of the AcrB trimer. AcrA and TolC are indicated in light green and light purple colors, respectively. (B) The lateral grooves in the L and T monomer indicate the substrate binding sites. The different geometric forms reflect low (triangle), high (rectangle), or no (circle) binding affinity for the transported substrates. In the first state of the cycle, a monomer binds a substrate (acridine) in its transmembrane domain (L conformation), subsequently transports the substrate from the transmembrane domain to the hydrophobic binding pocket (conversion to $\mathrm{T}$ conformation) and finally releases the substrate in the funnel toward TolC (O conformation). AcrA is postulated to participate in the transduction of the conformational changes from AcrB to TolC, which results in the opening of the TolC channel and the facilitation of drug extrusion to the outside of the cell. Adapted and modified from Seeger et al., 2006.

without ligand [70-72, 94, 95]. With one exception [72], most of these structures were derived from the first structure (PDB entry: 1IWG) taken as a template. In the years 2006 and 2007, 6 structures describing an asymmetric AcrB trimer were deposited (PDB entries: 2DHH, 2DR6, 2DRD, 2GIF, 2HRT, 2J8S)[67-69]. The best resolution structure (2.5 $\AA$, PDB entry 2J8S) was obtained with $\mathrm{AcrB} /$ designed ankyrin repeat proteins (DARPins) co-crystals [69]. Two of the deposited asymmetric structures were derived from 3.1 and 3.3 $\AA$ data of $A \mathrm{crB} / \mathrm{minocyclin}$ and $\mathrm{AcrB} /$ doxorubicin cocrystals, respectively [68]. From the asymmetric structures, a model for drug transport based on conformational cycling of the monomers by the RND pump AcrB has been proposed (Fig. 8) [67, 68].

\section{Conformational Cycling and Binding Change Mecha- nism}

Visual inspection of the periplasmic domain of the asymmetric AcrB structure (PDB entry: 2GIF) -viewed from the periplasmic side perpendicular to the membrane plane and direct comparison to the structure of the $\alpha / \beta$ subunits and $\gamma$ subunits of bovine $\mathrm{F}_{1} \mathrm{~F}_{\mathrm{o}}$ ATP synthase (PDB entry: 1BMF [96]) -viewed from the cytoplasm- leads to the overwhelm- ing impression that the structures are analogous (Fig. 9). The $\gamma$ rod of the ATPase is tilted towards one of the three $\alpha / \beta$ subunits. Likewise, in one monomer of the AcrB trimer the pore $\alpha$-helix ( $\mathrm{N} \alpha 2$, Fig. 6 ) is tilted towards the neighbouring monomer's PN2 subdomain. In the ATPase and AcrB structures, the monomers are in distinct different conformations, designated loose $(\mathrm{L})$, tight $(\mathrm{T})$ and open $(\mathrm{O})^{1}$. Long before structural details of the $\mathrm{F}_{1} \mathrm{~F}_{\mathrm{o}}$ ATP synthase were known, Paul Boyer postulated the binding change mechanism for this enzyme, describing a conformational cycling of the ( $\alpha$ and) $\beta$ subunits through the states loose, tight and open, leading to the synthesis of ATP [97-99].

One of the three key points of his binding change mechanism is that the energy input (i.e. proton motive force) is not used to form ATP, but to release the ATP molecule from the

\footnotetext{
' There is a marked difference between the designation of the $\alpha / \beta$ subunits of bovine $\mathrm{F}_{1} \mathrm{~F}_{\mathrm{o}}$ ATP synthase and that of the AcrB monomers. The $\gamma$ rod of the ATPase is tilted towards the "open" $\alpha / \beta$ subunits (despite appearances in Fig. 9), whereas the PN1 subdomain of the "open" monomer of AcrB is tilted towards the "tight" monomer. The functional relevance of the $\gamma$ rod of the ATPase as energy transducer is more likely compable with TM8 of AcrB. The coil to helix transition of TM8 in the AcrB "open" monomer appears to emphasize its role as energy transducer
} 
F1 ATPase, bovine mitochondria

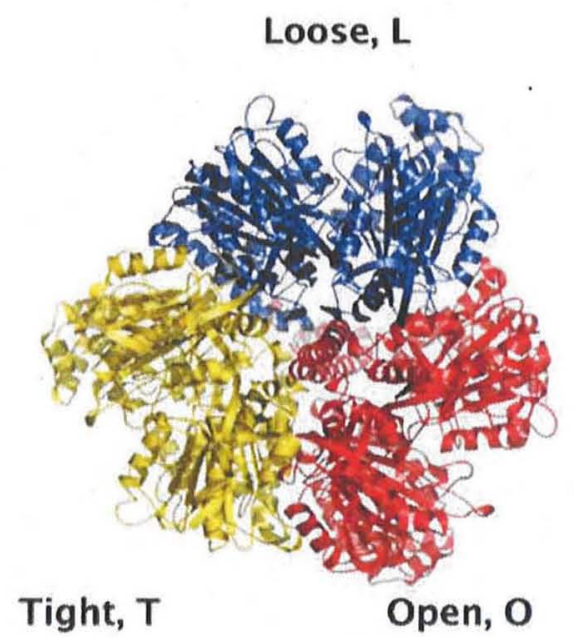

AcrB, Escherichia coli

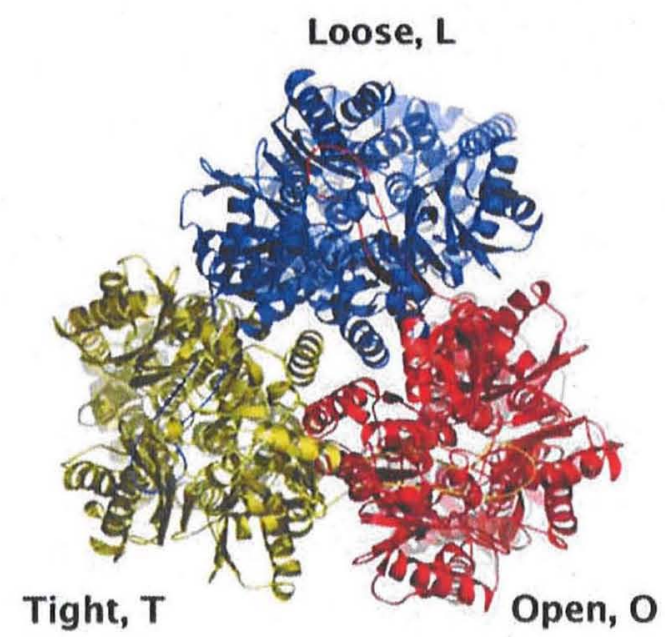

Fig. (9). Structural analogy between the $\alpha / \beta$ subunits and $\gamma$ subunits of bovine $\mathrm{F}_{1} \mathrm{~F}_{\mathrm{o}}$ ATP synthase (PDB entry: 1BMF, Abrahams et al., 1994) (left, viewed from the cytoplasm) and the periplasmic domain of the asymmetric AcrB structure (PDB entry: 2GIF, Seeger et al., 2006) (right, viewed from the periplasmic side perpendicular to the membrane plane). The structures are presented as ribbon diagrams and the designation of the individual monomers is indicated (Loose $(\mathrm{L})$, Tight $(\mathrm{T})$ and Open $(\mathrm{O})$ ). See text for details.

$\beta$ subunit. The second key point is the catalytic cooperativity: ATP can only be released when ADP and $\mathrm{P}_{i}$ are bound to another monomer within the hexameric $\mathrm{F}_{1}$ part of the ATP synthase (so called bi-site activation). The third key point is the rotational catalysis.

In analogy to the binding change mechanism (also called the alternating site mechanism) our group [67] and Murakami et al. [68] independently postulated an analogue alternating site functional rotation mechanism for the transport of drugs by AcrB (Fig. 8). The general concept describes a functional rotation starting with the (loose) binding of substrate to a low affinity site on the L monomer, followed by conformational change to the $\mathrm{T}$ conformer and tight binding of the substrate in the designated binding pocket and finally conversion to the $\mathrm{O}$ monomer resulting in the release of the substrate towards TolC. The $\mathrm{O}$ monomer finally converts to the $\mathrm{L}$ conformation restarting the cyclic event (Fig. 8). It has to be pointed out that the direction of events e.g. conversion from $\mathrm{L}$ to $\mathrm{T}, \mathrm{T}$ to $\mathrm{O}$, and back to $\mathrm{L}$ is based on interpretation of structural data only and that further experimental proof is needed to establish the consecutive conformational states. To date, three considerably different conformational states of the AcrB trimer have been crystallized: The "all-loose" or LLL conformation derived from diffraction data of crystals grown in the R32 space group [61], the asymmetric or LTO conformation obtained from crystals lacking the three-fold symmetry $[67,68]$ and the "all-tight" or TTT conformation (Eicher, Pos, unpublished). Moreover, cross-linking data supports the conformational flexibilty within the AcrB trimer to form the LLT, LTT and TTO conformations [78]. As is indicated in Fig. (10), these conformational states have been

incorporated into a more detailed scheme for drug transport, taking into account the cooperativity or bi-site activation, in analogy to the binding change mechanism by the $F_{1} F_{0}$ ATP synthase. In the proposed scheme (Fig. 10), the LLL conformation of the AcrB trimer describes a state when the pro- tein is depleted from substrate ("resting state"[94]). Binding of substrate to the $\mathrm{L}$ monomer results in the conversion from $\mathrm{L}$ to $\mathrm{T}$ and, according to the binding change mechanism, a second substrate has to bind to another monomer as a prerequisite for the release of the substrate to the outside i.e. substrate has to bind to another monomer before conversion of the substrate-occupied $\mathrm{T}$ monomer to the $\mathrm{O}$ monomer can occur (Fig. 10). The conformational change from the $T$ to the $\mathrm{O}$ monomer is proposed to be an energy dependent step, in analogy with the energy dependence of the release of ATP from the $\beta$-subunit. Proton uptake from the periplasm is anticipated in this step (Fig. 10). Structurally, the conversion of the $\mathrm{T}$ monomer to the $\mathrm{O}$ monomer might be sterically facilitated by the formation of the adjacent T monomer (Figs. 10 and 11). The conversion from the $\mathrm{L}$ monomer to the $\mathrm{T}$ monomer comprises substantial movement of the PN2 subdomain (LTO to TTO). This causes the loss of restraints for the adjacent PN1 subdomain of the already existent T monomer from the LTO trimer and facilitates its pronounced inclination towards the other T monomer (Fig. 11) [67, 68]. Its conversion to the $\mathrm{O}$ monomer follows the conversion from TTO to LTT. The combined effect of binding of a second substrate to the AcrB trimer (leading to the formation of a second $\mathrm{T}$ monomer) with the binding of (a) proton(s) to the transmembrane domain (driven by the proton motive force), results in the formation of the $\mathrm{O}$ monomer.

The basis for the conversion of the $\mathrm{O}$ monomer to the $\mathrm{L}$ monomer (TTO to LTT) is still unclear (Fig. 10). However, two neighbouring $\mathrm{O}$ monomers would cause an intermonomeric steric clash between the PN1 and PN2 subdomains like has been shown for the $\mathrm{O}$ and $\mathrm{L}$ monomer (Fig. 11).

Binding of a third substrate to the L conformer of an LTT AcrB trimer (Fig. 10) is considered to be possible (e.g. in the case of high substrate concentration) and will trigger the creation of the third T monomer resulting in the "all-tight" conformation (TTT), a conformation found in AcrB crystals 


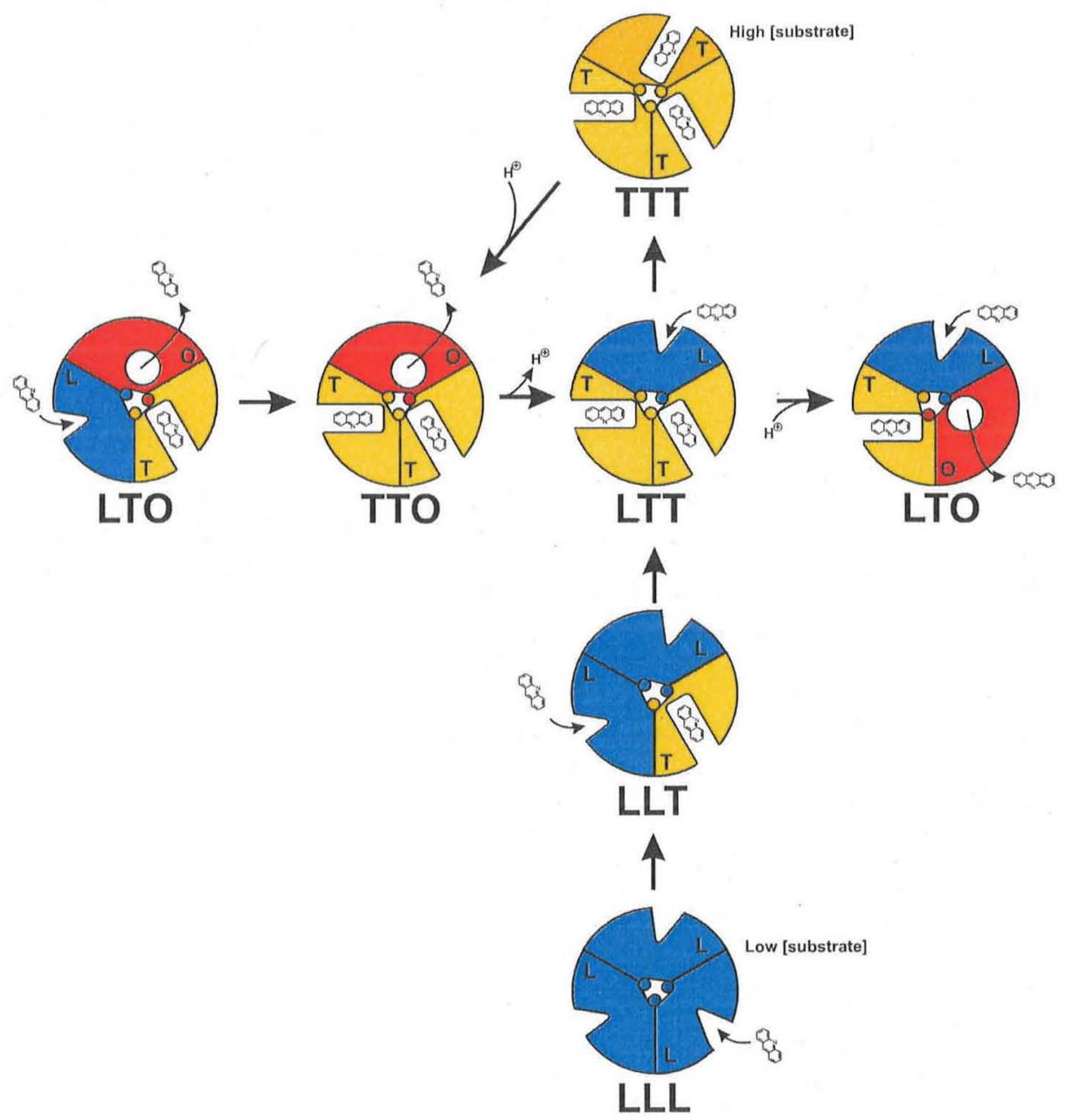

Fig. (10). Schematic representation of the AcrB alternating site functional rotation transport mechanism extended by postulated intermediate steps. The conformational states loose $(\mathrm{L})$, tight $(\mathrm{T})$, and open $(\mathrm{O})$ are colored blue, yellow and red, respectively. The lateral grooves in the $\mathrm{L}$ and $\mathrm{T}$ monomer indicate the substrate binding sites. The different geometric forms reflect low (triangle), high (rectangle), or no (circle) binding affinity for the transported substrates. Both states LTO at the far left and far right are identical to the states shown in Fig. (8). State TTT is postulated to occur at high substrate concentration. The states LLL and LLT are postulated to occur in the absence or at low substrate concentrations. See text for details.

(Eicher, Pos et al., unpublished). Energy input from the proton motive force leads to proton binding to the transmembrane domain of one of the $\mathrm{T}$ monomers, formation of the $\mathrm{O}$ monomer and finally conversion to the $\mathrm{L}$ monomer. Interestingly, cross-link data supports the formation of more than one of the same conformer (TTL and LLT) in E. coli membranes [78], whereas in crystallization experiments the formation of symmetric forms of the AcrB trimer (LLL, TTT) appears to be predominant to the formation of asymmetric AcrB trimers in crystals. For the crystalline state, this may be explained by the reduced free energy of symmetric crystal contacts as compared to asymmetric ones.

\section{Tunnels Guiding the Substrate}

Upon examination for cavities and tunnels in the asymmetric AcrB structure [67, 69] (PDB entries: 2GIF, 2JS8) a tunnel system leading to and away from the hydrophobic substrate binding pocket became apparent (Fig. 12). The L monomer (Fig. 12A, blue) contains a tunnel (designated tunnel 2) starting at the lateral cleft (subdomain PC1/PC2 interface) about $15 \AA$ above the putative membrane plane and protruding into the porter domain. In the T monomer (Fig. $12 \mathrm{~A}$, yellow), the formation of an additional tunnel (tunnel 1) in the porter domain is apparent with its entrance located at height of the TM8 and TM9 groove, which has been postulated to accommodate substrates originating from the membrane [61, 67-69]. Indeed, good resolution (2.5 $\AA$ ) data [69] shows the presence of a dodecylmaltoside molecule (which is both detergent and AcrB substrate) located in the TM8/TM9 groove of the T monomer. Tunnel 1 merges with tunnel 2 close to the hydrophobic substrate binding pocket (Fig. 12, inset with bound minocyclin). In the $\mathrm{O}$ monomer (Fig 12B, red), the lateral opening and tunnels 1 and 2 are absent due to the closure of the lateral cleft caused by the 


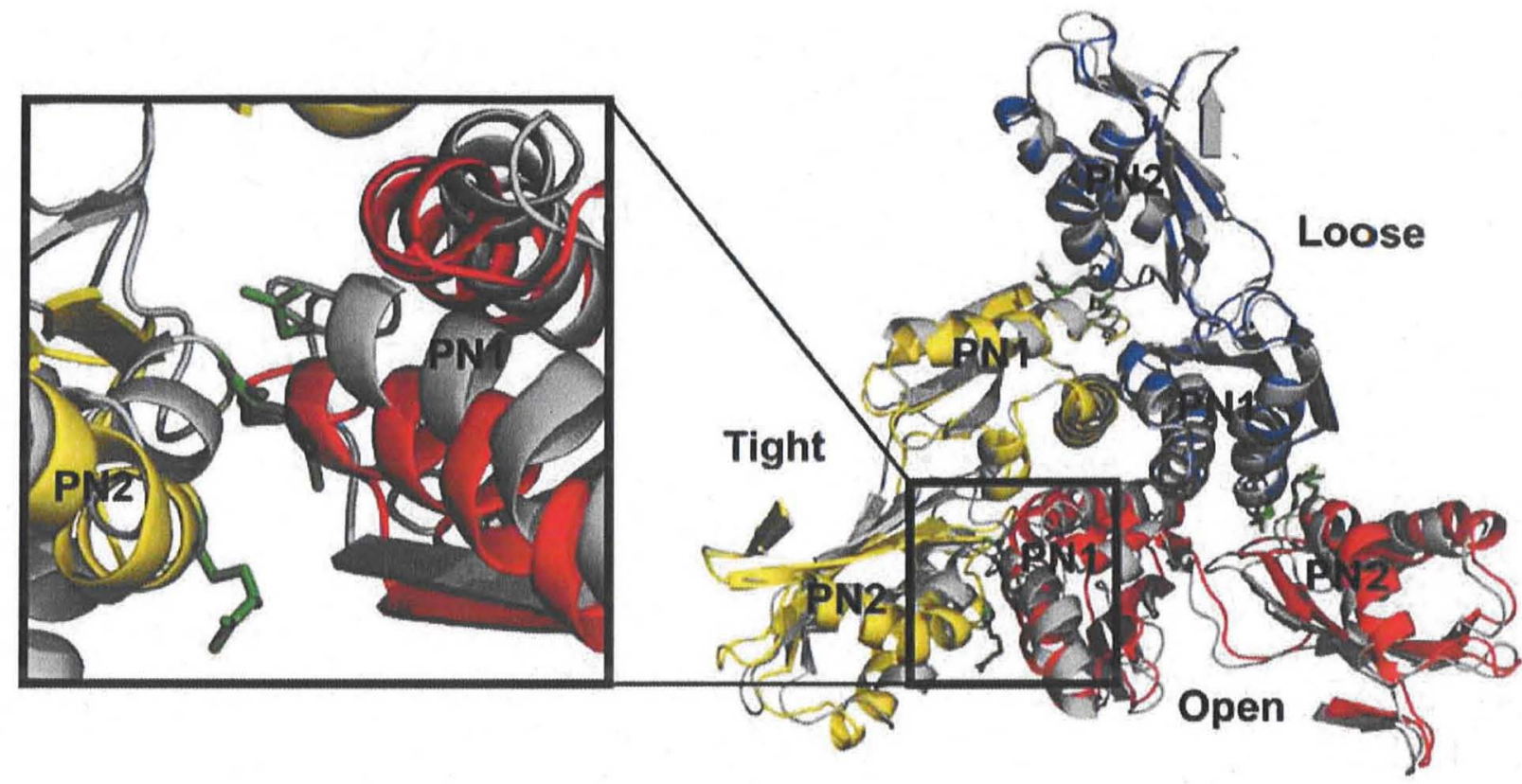

Fig. (11). Main structural differences of the PN1 and PN2 subdomains in the AcrB trimer. Right: The three AcrB PN1 and PN2 subdomains of the $\mathrm{L}$ (in blue), $\mathrm{T}$ (yellow) and $\mathrm{O}$ (red) monomers in top view as ribbon presentation are superimposed onto the PN1 and PN2 subdomains of the symmetric AcrB trimer model depicted in gray. Inset (on the left): The PN1 subdomain of the O monomer would sterically clash with the PN2 subdomain of a neighbouring L monomer (in grey). Conversion of the $\mathrm{L}$ monomer (grey) into the T monomer (yellow) would allow movement of the PN1 subdomain of the $\mathrm{O}$ monomer without steric hindrance.

large conformational change of the PC2 subdomain and the coil to helix transition of TM8 during the T to $\mathrm{O}$ monomer transition (Fig. 12B) [67,68]. In the $\mathrm{O}$ monomer, tilting of the central helix $(\mathrm{N} \alpha 2)$ due to the movement of the PN1 subdomain creates an exit pathway (tunnel 3, Fig. 12B) which leads to the funnel of the AcrB trimer. In a conformational cycling scenario, the tunnels form an alternate access pathway between the TM8/TM9 groove (tunnel 1) or/and the lateral side (tunnel 2) of the AcrB trimer and tunnel 3 towards the funnel and TolC (Fig. 12) [67, 69]. In addition, we postulated a peristaltic mode of drug transport through the tunnels, actively guiding the substrates from the periplasmic space/membrane towards TolC and the outside of the cell [67].

\section{Substrate Transport Through the Tunnel System}

The lateral access of tunnel 2 is located $15 \AA$ above the putative membrane plane (Fig. 12A) and at first sight appears to be suitable for the collection of substrates residing in the periplasmic space, i.e. the $\beta$-lactams. However, resistance against $\beta$-lactams due to the action of the AcrAB-TolC system of Salmonella typhimurium (AcrB of $S$. typhimurium shares $94 \%$ identical residues with AcrB of $E$. coli) was positively correlated with the increment in the penicillin side chain octanol-water partition coefficient, the preferred substrates for the pump being nafcillin, cloxacillin and benzylpenicillin [29]. The hydrophobic side chains of these amphiphilic substrates are likely to submerge into the outer leaflet of the inner membrane, whereas the $\beta$-lactam ring would remain in the periplasm. The outer leaflet localization makes these compounds suitable candidates for entering the
AcrB tunnel 1 via the TM8/TM9 groove. It remains elusive whether other substrates like e.g. fluoroquinolones and macrolides are also garnered from the membrane or whether tunnel 1 can be considered the general entrance tunnel for all substrates. The role of tunnel 2 remains elusive as well. The lateral opening of tunnel 2 is located at the PC1/PC2 cleft, which was postulated to be an interaction site for AcrA [61, 91]. The latter might function as a flexible lid and might alternately open and shield the entrance towards the periplasm. Moreover, an alternative hypothesis would be that tunnel 2 rather functions as an exit tunnel for non-substrates to leave the tunnel system (Fig. 12). The porter domain of AcrB and its homologues is responsible for substrate selectivity [7375](see above), which is in complete accordance to the localization of the substrates minocyclin and doxorubicin in the hydrophobic pocket of the T monomer (Fig. 12 inset) (Eicher, Pos et al., unpublished) and [68]. Therefore, substrate binding occurs in the porter domain, where the hydrophobic pocket acts as a selectivity filter $[67,68]$. The results from the periplasmic domain swapping experiments [73-75] imply that selection takes place not until in the porter domain and suggest that non-substrates are also transported from the transmembrane domain towards the binding pocket. Deselected compounds have to leave the porter domain either via back-diffusion through tunnel 1, but might also be bypassed through tunnel 2 (Fig. 12). Residues lining tunnel 1 and 2 are charged and/or polar rather than hydrophobic. To allow transport through the rather hydrophilic tunnel(s), a peristaltic transport mechanism would account for unidirectionality of substrate transport and the movement of hydrophobic compounds through rather hydrophilic tunnels. 


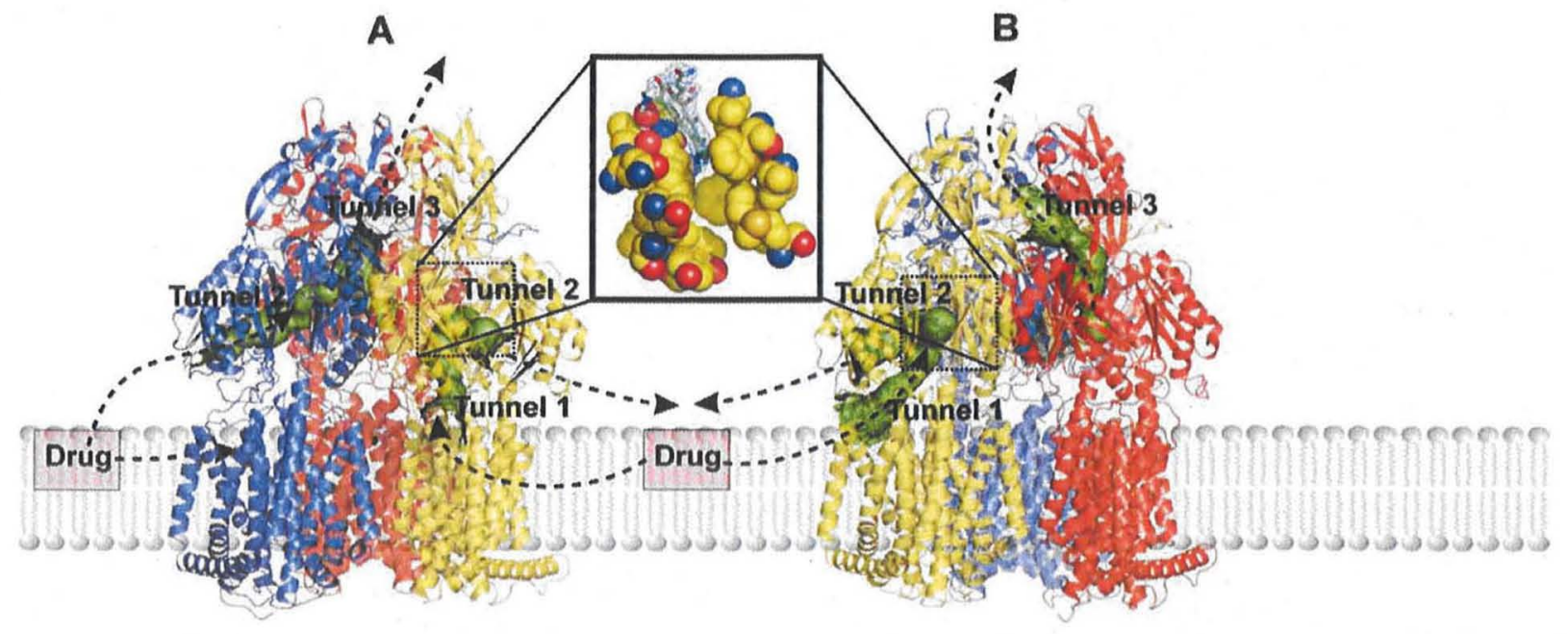

Fig. (12). Visualization of tunnels in the porter (pore) domain of the trimeric AcrB peristaltic drug efflux pump. The AcrB monomers are presented in (A) blue (loose, L), (B) yellow (tight, T) and (c) red (open, O). The tunnels are highlighted as green surfaces in a ribbon model of the AcrB trimer. Inset: In the T monomer (yellow), a hydrophobic pocket is defined by phenylalanines 136, 178, 610,615, 617, and 628; valines 139 and 612; isoleucines 277 and 626; and tyrosine 327 at the PN2/PC1 interface. Bound minocyclin is depicted with the observed electron density in a $2 \mathrm{Fo}-\mathrm{Fc}$ electron density map countoured $1 \sigma$ (Seeger, Pos et al., unpublished). Panels A and B represent in each case a one third conversion of a full $\mathrm{L} \rightarrow \mathrm{T} \rightarrow \mathrm{O} \rightarrow \mathrm{L}$ cycle.

Once substrate is recognized and bound to the binding pocket in the $\mathrm{T}$ monomer, conformational change is triggered and leads to conversion from the $\mathrm{T}$ monomer to the $\mathrm{O}$ monomer (Figs. 8, 10 and 12). However, as stated above, binding of substrate is most likely not the exclusive trigger for conformational change from $\mathrm{T}$ to $\mathrm{O}$. In accordance to the binding change mechanism theory for the ATP synthase, bound substrate might not be released before a second substrate binds to another monomer i.e. the adjacent $\mathrm{L}$ monomer (Fig. 10). In this context, it is postulated for AcrB that only if the trimer is in a LTT (or possibly TTT) conformation, release of the substrate and conformational change to LTO (or possibly via TTO and LTT) occurs. It appears that detergent solubilized AcrB is preferably present in its asymmetric conformation, as is implied from cross-linking [77, 78] and DARPin binding [69]. Notwithstanding, AcrB readily crystallizes at the "all-loose" symmetric form [61, 63, 70-72, 94, 95] and also in its "all-tight" state (Eicher, Pos et al. unpublished) in a wide range of crystallization conditions, whereas the asymmetric LTO conformation was only retrieved at a more defined crystallization condition. The formation of crystals containing AcrB in different conformational states shows the flexibility of this membrane protein to adopt intermediate cycling states.

The AcrB structure derived from AcrB/DARPin co-crystals diffracting to $2.5 \AA$ (PDB entry: $2 \mathrm{~J} 8 \mathrm{~S}$ ) showed very low RMSD values compared to the 2.9-3.0 $\AA$ structures derived from our group (PDB entries: 2GIF, 2HRT), indicating near identity of the structures [69]. Interestingly, the $2.9 \AA$ AcrB structure (PDB entry: 2GIF) [67] only discloses tunnel 2 in the $\mathrm{L}$ and $\mathrm{T}$ monomer [67], whereas in the $2.5 \AA \mathrm{AcrB} /$ DARPin co-crystal structure [69] both tunnel 1 and 2 are apparent in the T monomer (as shown in Fig. 12). Moreover, the only striking difference between the 2J8S and 2GIF structures is the side chain orientation of Phe563 in the
TM8/TM9 region (Fig. 13). The cavity and tunnel algorithms CAVER [100] and MOLE [101] were able to find the tunnel 1 entrance in $2 \mathrm{~J} 8 \mathrm{~S}$, but indicated its absence in $2 \mathrm{GIF}$.

A possible interpretation is that the AcrB/DARPin cocrystal structure might represent another intermediate state exemplifying the structural flexibility of AcrB. As has been shown very recently, flexibility and the formation of intermediate states appear to be very common for the symmetric AcrB trimer [71, 72, 94]. The extent of the conformational differences between the published symmetric AcrB forms is small, whereas the monomers within the asymmetric trimer show substantial conformational differences.

The suggested path of the substrate does not yet consider a role for prominent features such as the vestibules [61] and the central cavity (Fig. 5A,D). Recent structural studies [70$72,94,95]$ are, however, indicative for an implication of the central cavity on drug binding and transport. Careful analysis of this structural data derived from R32 crystals e.g on twinning and more distinct substrate assignment would be helpful to substantiate the role of the cavity on substrate binding. Substrate binding studies with isolated detergent soluble AcrB from $E$, coli indicated drug binding affinities between 5.5 and $74 \mu \mathrm{M}$ [102] comparable to drug binding affinities of MdfA (MF Superfamily) [103] and EmrE (SMR Superfamily) [104]. The results suggested a drug to AcrB monomer stoichiometry of 1 , which is in agreement with the proposed binding of substrates in the cavity [70], but stands in contrast to the drug to AcrB trimer stoichiometry of 1 found in the asymmetric AcrB structure [68].

\section{Transport of Protons and Energy Transduction}

Transport of drugs from the cell to the outside by the AcrA/AcrB/TolC system is coupled to proton transport from the periplasm to the cytoplasm. Proton binding and release 


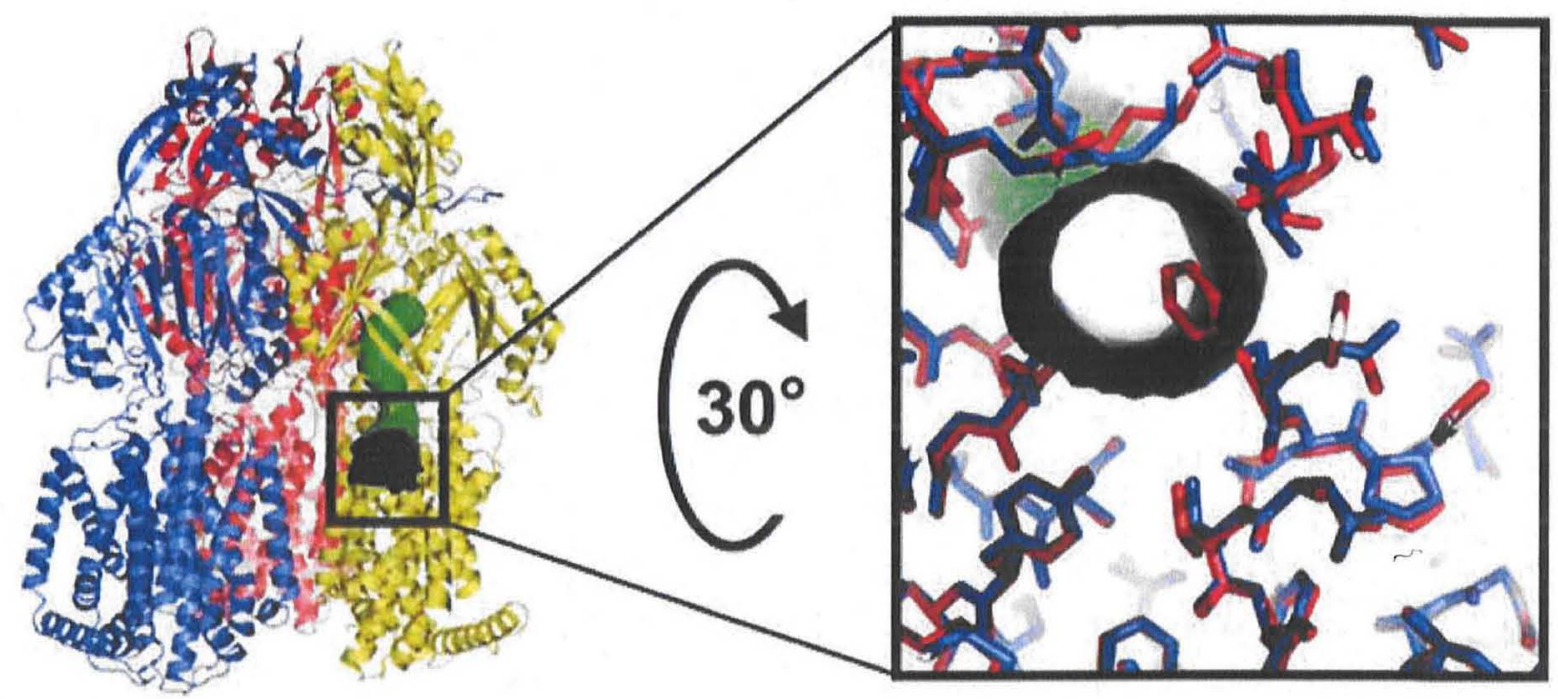

Fig. (13). Location of the potential tunnel 1 gating residue Phe563 in the T monomer of the AcrB trimer. Inset: Superimposition of AcrB trimer structures 2J8S (in blue) and 2GIF (in red). The side chain orientation of Phe563 in the 2GIF structure prevents detection of tunnel 1 and putative transport from the TM8/TM9 groove towards the binding pocket in the T monomer.

takes place in the transmembrane domain, where key residues involved in proton transport have been identified by mutagenesis, functional assays and studying the asymmetric AcrB structure.

Single AcrB mutants where D407, D408, K940, T978 or R971 were substituted by alanine caused complete loss of function of the tripartite efflux system $[65,66]$. In the asymmetric structure [67-69], K940 and R971 (and to some extent also D407) have distinct different side chain conformations when comparing the $\mathrm{L}$ and $\mathrm{T}$ monomers with the $\mathrm{O}$ monomer (Fig. 14A,B). The subtle side chain reorientations observed in the $\mathrm{O}$ monomer appear to be leveraged via TM8 to the periplasmic s ubdomains $\mathrm{PC} 2$ and $\mathrm{PN} 1$ where large rigid body conformational changes appear to have taken place (Fig. 14A,B). The PC2 and PN1 movement leads to closure of tunnels 1 and 2 (and the substrate binding pocket) and opening of tunnel 3 towards the funnel (Fig. 14C). Moreover, the periplasmic loop connecting TM3 and TM4 is downshifted approx. $3 \AA$ towards the membrane during the T to $\mathrm{O}$ monomer transition and appears to relieve constraints for the PN1 domain to adopt its inclined conformation as described above. The exact chain of events from proton binding towards coil-to-helix transition of TM8 (Fig. 14B) and the apparent downshift of the transmembrane domain during $\mathrm{T}$ to $\mathrm{O}$ monomer conformational change is not completely clear from the structure per se $[67-69,94]$ and needs further investigation.

From the local environment of the D407, D408, K940 and R971 side chains in the different monomers, the protonation state of these side chains can be assessed. As AcrB is energized by the proton-motive force, transient protonation of titratable groups within the transmembrane domain of the protein can be expected to be the mechanism which delivers the energy required for the conformational changes described above. A prominent K940 (TM10) side chain reorientation away from D408 and towards D407 (both on TM4) in the O conformation (Fig. 14C, lower panel), and a bulging of TM5 towards TM4 and TM10 [67] is indicative of protonation and deprotonation events. As stated above, the conversion of the $\mathrm{T}$ monomer to the $\mathrm{O}$ monomer is postulated to be the energydependent step in accordance with the ATP synthase binding change mechanism. Hence, proton uptake from the periplasm is expected in the T monomer. Further uptake of protons from the periplasm and subsequent release to the cytoplasm in any of the monomers is anticipated but cannot be elucidated from the structural information up to date. High resolution data of $\mathrm{AcrB}$ in its asymmetric conformation to locate structural water molecules in the transmembrane domain, as well as further biochemical data are necessary to obtain more certainty about the chain of protonation/deprotonation events.

\section{Stoichiometry of Drug/Proton Antiport}

To date there are only a few reports on in vitro assays using reconstitution of the inner membrane RND component (AcrB/AcrD) into liposomes $[79,80]$. Reconstitution of activity was dependent on addition of the soluble form of the MFP component (AcrA) to AcrB or AcrD containing proteoliposomes. Despite the succes of retrieving AcrB and AcrD activity outside the native tripartite setup, readout is still not suitable for quantification of the drug/proton antiport activity. Transport has been shown to be dependent on $\Delta \mathrm{pH}$ and assumed to be dependent on $\Delta \psi$ as well. Considering the wide variety of substrates (anionic, cationic neutral) stoichiometric prediction become highly complicated and suggests coupling flexibility of the drug pump system as has been shown for MdfA [105]. As has been strongly advocated by Nikaido and colleagues $[29,80,106]$ and is supported by the latest AcrB structures [61, 67-69], substrates might be in general collected from the outer leaflet of the inner membrane, where they most probably are at a high concentration compared to the periplasm/cytoplasm. The question on electrogenicity of the system would then become obsolete, since substrates would not be transported across a membrane. Hence, the coupling proton(s) transported from the periplasm 


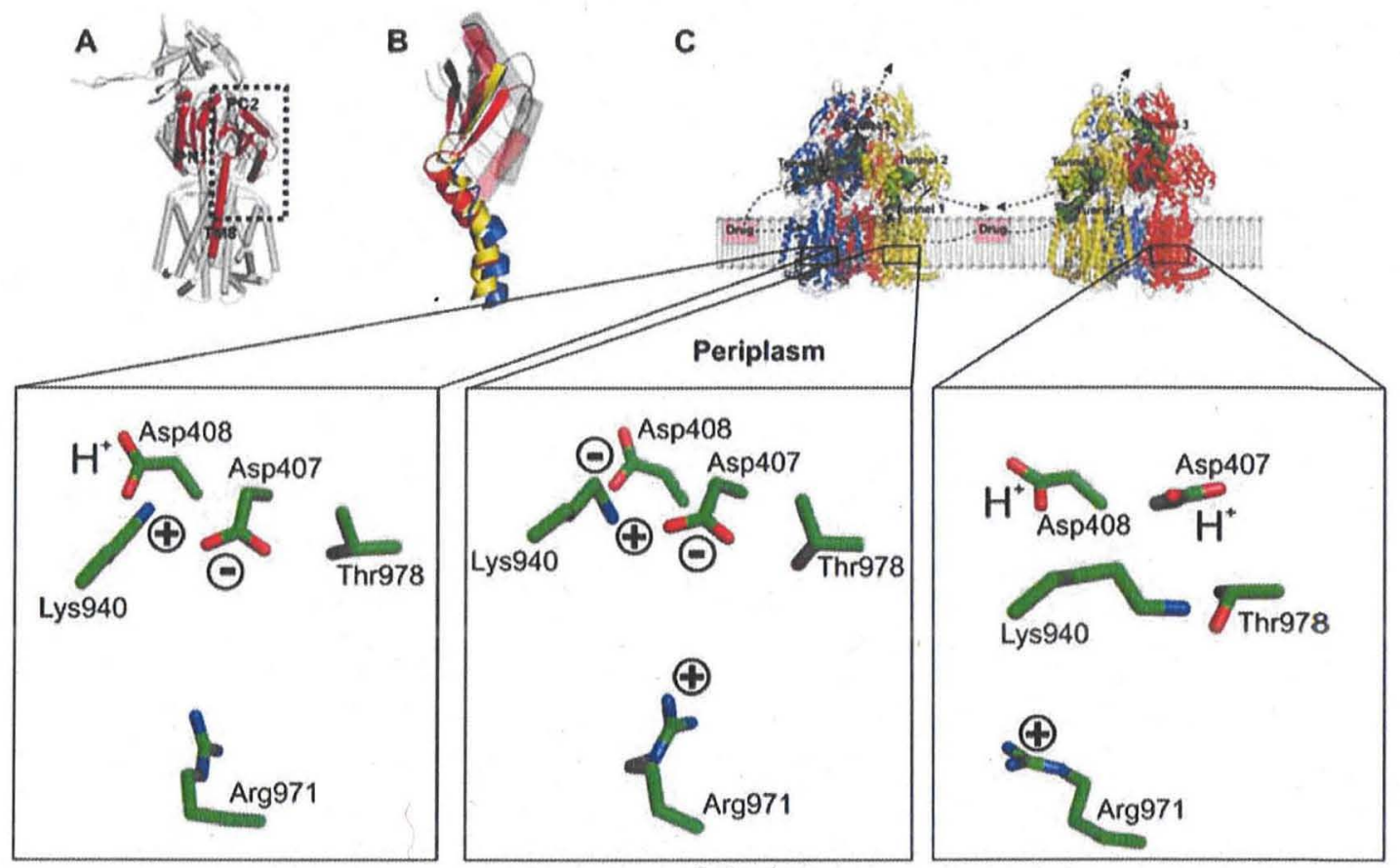

Cytoplasm

Fig. (14). (A) Side view superimposition of the AcrB L monomer (grey) and the PN1, PC2 subdomains as well as TM8 of the $\mathrm{O}$ monomer (red). (B) In a close-up view of the boxed region of (A) the N-terminal part of TM8 (residues 859 to 880 ) and the PC2 subdomain (residues 679-721 and 822-858) are superimposed. The structures in blue, yellow and red represent the conformations of the TM8 and the C-terminal $\beta$-sheets (C $\beta 15)$ of the PC2 subdomains of the $\mathrm{L}, \mathrm{T}$ and $\mathrm{O}$ monomers, respectively. The rest of the PC2 subdomain is depicted in transparent grey (L and T monomers) or red (O monomer) (C) Side view representation of the AcrB trimer as shown in Fig. (12). The boxed region of the transmembrane domains comprising the essential residues Asp407, Asp408, Lys940, Arg971 and Thr978 of the L, T and O monomers are shown in a close-up view. Proton uptake is anticipated in the T monomer (middle box) and is postulated to lead to the side chain reorientation of Asp407, Lys940 and Arg971. These side chain conformational changes might be coupled to the coil-to-helix transition shown in (B) and the PN1/PC2 subdomain movement as shown in (A)

to the inside of the cell by AcrB would define the overall mechanism electrogenic.

\section{AcrA/AcrB/TolC Drug Transport Hypothesis}

A hypothesis on drug transport by the $\mathrm{Acr} A / \mathrm{AcrB} / \mathrm{TolC}$ three component system is depicted in Fig. (15). Aside from the conformational signal transduction from AcrB to TolC via the AcrA linker $[67,68,82,84,88,91]$ (B. Luisi and colleagues in this issue), there is a line up of drug molecules from the AcrB funnel continuing into the TolC channel. The latter postulate results from two observations: 1) Residues lining the observed AcrB tunnels and the TolC channel are polar rather than hydrophobic. 2) The TolC channel is recruited by other systems to extrude a wide variety of compounds including polypeptides as in the $E$. coli type I extrusion system HlyB/HlyD/TolC transporting HlyA (haemolysin). The $A B C$ transporter HlyB is energized by the free energy of hydrolysis of ATP to drive HlyA transport to the medium. The export process is dependent on HlyD, a member of the MFP family and TolC. It seems plausible that
HlyA extrusion is reliant on a mechanical (maybe peristaltic) movement through the transport system rather than diffusion. Despite the lack of covalent bonds between the separate AcrB substrate molecules, the mode of transport by a mechanical, peristaltic movement of a chain of substrates would account for strict unidirectional movement towards the outside of the cell.

The last decade has brought us an enormous leap in understanding on how the AcrA/AcrB/TolC and homologue systems transport drugs and other compounds across the inner and outer membrane of the Gram-negative cell. Despite the wealth of the recent structural and functional data on all components of the tripartite system, we still lack the basic understanding on drug/proton coupling and stoichiometry, energy transduction, drug binding and transport kinetics.

\section{ACKNOWLEDGEMENTS}

This review includes many fine ideas of scientist whom I (KMP) had the pleasure to discuss transport mechanisms in 


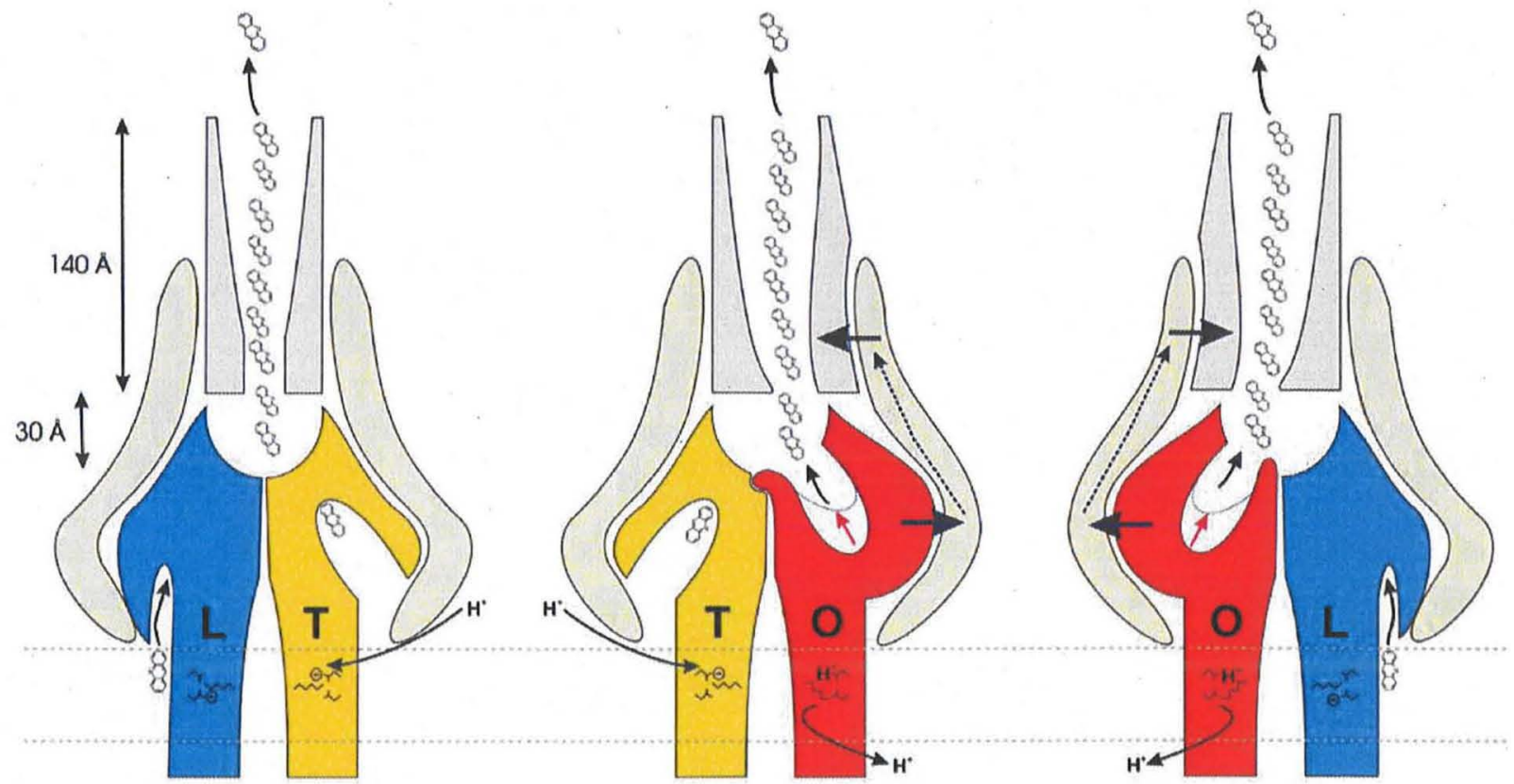

Fig. (15). Schematic representation of the AcrB alternating site functional rotation transport mechanism. The conformational states loose (L), tight $(\mathrm{T})$, and open $(\mathrm{O})$ are colored blue, yellow and red, respectively. Only two of the three monomers of the AcrB trimer are shown in sideview. AcrA and TolC are indicated in light green and grey, respectively. The proposed proton translocation site (D407, D408, and K940) is indicated in the membrane part of each monomer. In the first state of the cycle (from left to right), a monomer binds a substrate (acridine) in its transmembrane domain ( $\mathrm{L}$ conformation), subsequently transports the substrate from the transmembrane domain to the hydrophobic binding pocket (conversion to $\mathrm{T}$ conformation) and finally releases the substrate in the funnel toward TolC (O conformation). Peristaltic transport of drugs through the AcrB tunnels (indicated by the red arrow) and through TolC in combination to the line up of drug molecules inside the AcrB funnel and the TolC channel would account for strict unidirectional movement towards the outside of the cell. The conversion from the $\mathrm{T}$ monomer to the $\mathrm{O}$ monomer conformation is suggested to be the major energy-requiring (proton motive force-dependent) step in this functional rotation cycle and requires the binding of a proton to the proton translocation site (D407, D408, and K940) from the periplasm. The release of a proton from the proton translocation site to the cytoplasm might occur during conversion from the $\mathrm{O}$ monomer to the $\mathrm{L}$ monomer or from the latter to the T monomer. AcrA is expected to participate in the transduction of the conformational changes from AcrB to TolC (indicated by black arrows), which results in movement of the proximal part of TolC and the facilitation of drug extrusion to the outside of the cell.

general and of AcrB in particular. I like to thank S. Murakami, E. Bibi, S. Schuldiner, K. Locher, D. Picot, D. Mueller, C. Tate, B. Luisi, R. Friedman, H. van Veen, G. Sennhauser, P. Amstutz, M. Grütter, J. Bohnert, O. Lomovskaya and many others for their open discussion philosophy.

\section{ABBREVIATIONS}

$\begin{array}{ll}\text { Acr } & =\text { Acriflavine resistance } \\ \text { AcrB } & =\text { Acriflavine resistance protein } \mathrm{B} \\ \mathrm{L} \text { monomer } & =\text { Loose monomer (access monomer) } \\ \mathrm{T} \text { monomer } & =\text { Tight monomer (binding monomer) } \\ \mathrm{O} \text { monomer } & =\text { Open monomer (extrusion monomer) } \\ \mathrm{MDR} & =\text { Multidrug resistance } \\ \mathrm{OM} & =\text { Outer Membrane } \\ \mathrm{IM} & =\text { Inner Membrane } \\ \mathrm{MIC} & =\text { Minimal Inhibitory Concentration }\end{array}$

$\begin{array}{ll}\text { RMSD } & =\text { Root mean square distance } \\ \text { PDB } & =\text { Protein Data Bank }\end{array}$

\section{REFERENCES}

[1] Saier, M. H., Jr. (2000) Microbiol. Mol. Biol. Rev., 64, 354-411. [2] Saier, M. H., Jr. and Paulsen, I. T. (2001) Semin. Cell Dev. Biol., 12, 205-13.

[3] Bolhuis, H.; van Veen, H. W.; Molenaar, D.; Poolman, B.; Driessen, A. J. and Konings, W. N. (1996) EMBO J., 15, 4239-45.

[4] Dawson, R. J.and Locher, K. P. (2006) Nature, 443, 180-5

[5] Edgar, R. and Bibi, E. (1997) J. Bacteriol., 179, 2274-80.

[6] Yin, Y.; He, X.; Szewczyk, P.; Nguyen, T. and Chang, G. (2006) Science, 312, 741-4.

[7] Abramson, J.; Smirnova, I.; Kasho, V.; Verner, G.; Kaback, H. R and Iwata, S. (2003) Science, 301, 610-5.

[8] Huang, Y.; Lemieux, M. J.; Song, J.; Auer, M. and Wang, D. N. (2003) Science, 301, 616-20.

[9] Schuldiner, S.; Granot, D.; Mordoch, S. S.; Ninio, S.; Rotem, D.; Soskin, M.; Tate, C. G. and Yerushalmi, H. (2001) News Physiol. Sci., 16, 130-4.

[10] Tate, C. G.; Ubarretxena-Belandia, I. and Baldwin, J. M. (2003) J. Mol. Biol., 332, 229-42.

[11] Otsuka, M; Yasuda, M.; Morita, Y.; Otsuka, C.; Tsuchiya, T.; Omote, H. and Moriyama, Y. (2005) J. Bacteriol., 187, 1552-8. 
[12] Goldberg, M.; Pribyl, T.; Juhnke, S. and Nies, D. H. (1999) J. Biol. Chem., 274, 26065-70.

[13] Murakami, S. and Yamaguchi, A. (2003) Curr. Opin. Struct. Biol., 13, 443-52.

[14] Poole, K (2001) J Mol Microbiol. Biotechnol., 3 255-64.

[15] Ohgami, N.; Ko, D. C.; Thomas, M.; Scott, M. P.; Chang, C. C. and Chang, T. Y. (2004) Proc. Natl. Acad. Sci. USA, 101, 12473-8.

[16] Fralick, J. A. (1996) J. Bacteriol., 178, 5803-5.

[17] Hirakawa, H.; Nishino, K.; Yamada, J.; Hirata, T. and Yamaguchi, A. (2003) J. Antimicrob. Chemother, 52, 576-82.

[18] Sulavik, M. C.; Houseweart, C.; Cramer, C.; Jiwani, N.; Murgolo, N.; Greene, J.; DiDomenico, B.; Shaw, K. J.; Miller, G. H.; Hare, R. and Shimer, G. (2001) Antimicrob. Agents Chemother, 45 1126-36

[19] Kobayashi, N.; Nishino, K. and Yamaguchi, A. (2001) J. Bacteriol., 183, 5639-44.

[20] Nakamura, H. (1965) J. Bacteriol., 90, 8-14

[21] Ma, D.; Cook, D. N.; Alberti, M.; Pon, N. G.; Nikaido, H. and Hearst, J. E. (1993) J. Bacteriol., 175, 6299-313.

[22] Elkins, C. A.and Nikaido, H. (2003) J. Bacteriol., 185, 5349-56.

[23] Baranova, N. and Nikaido, H. (2002) J. Bacteriol., 184, 4168-76.

[24] Nagakubo, S.; Nishino, K.; Hirata, T. and Yamaguchi, A. (2002) J. Bacteriol., 184, 4161-7.

[25] Kobayashi, K,; Tsukagoshi, N. and Aono, R. (2001) J. Bacteriol. 183, 2646-53.

[26] Poole, K.; Krebes, K; McNally, C. and Neshat, S. (1993) J. Bacteriol., 175, 7363-72

[27] Poole, K. (2004) Clin. Microbiol. Infect, 10, 12-26.

[28] Nikaido, H. (1996) J. Bacteriol., 178, 5853-9.

[29] Nikaido, H.; Basina, M.; Nguyen, V. and Rosenberg, E. Y. (1998) J. Bacteriol, 180, 4686-92.

[30] Li, X. Z.; Zhang, L. and Poole, K. (1998) J. Bacteriol., 180, $2987-$ 91.

[31] Thanassi, D. G.; Cheng, L. W. and Nikaido, H. (1997) J. Bacteriol., $179,2512-8$

[32] Kawamura-Sato, K.; Shibayama, K.; Horii, T.; Iimuma, Y.; Arakawa, Y. and Ohta, M. (1999) FEMS Microbiol. Lett., 179, 345-52.

[33] Lau, S. Y. and Zgurskaya, H. I. (2005) J. Bacteriol., 187, 7815-25.

[34] Rahmati, S.; Yang, S.; Davidson, A. L. and Zechiedrich, E. L. (2002) Mol. Microbiol., 43, 677-85

[35] Lomovskaya, O.; Warren, M. S.; Lee, A.; Galazzo, J.; Fronko, R.; Lee, M.; Blais, J.; Cho, D.; Chamberland, S.; Renau, T.; Leger, R. Hecker, S: Watkins, W.; Hoshino, K. Ishida, H. and Lee, V J. (2001) Antimicrob. Agents Chemother., 45, 105-16.

[36] Bohnert, J. A. and Kern, W. V. (2005) Antimicrob. Agents Chemother., 49, 849-52

[37] Levy, S. B. and McMurry, L. (1978) Nature, 276, 90-2

[38] de Cristobal, R. E.; Vincent, P. A. and Salomon, R. A. (2006) J. Antimicrob. Chemother, 58, 31-6.

[39] Piddock, L. J. (2006) Nat. Rev. Microbiol., 4, 629-36.

[40] Piddock, L. J. (2006) Clin. Microbiol. Rev., 19, 382-402.

[41] Poole, K. (2000) Antimicrob. Agents Chemother., 44, $2595-9$.

[42] Grkovic, S.; Brown, M. H. and Skurray, R. A. (2002) Microbiol Mol. Biol. Rev., 66, 671-701, table of contents.

[43] Nagel de Zwaig, R. and Luria, S. E. (1967) J. Bacteriol., 94, 1112 23.

[44] Zakharov, S. D.; Eroukova, V. Y.; Rokitskaya, T. I.; Zhalnina, M V.; Sharma, O.; Loll, P. J.; Zgurskaya, H. I.; Antonenko, Y. N. and Cramer, W. A. (2004) Biophys. J., 87, 3901-11.

[45] Thanabalu, T.; Koronakis, E.; Hughes, C. and Koronakis, V. (1998) EMBO J. $17,6487-96$

[46] Koronakis, V.; Sharff, A.; Koronakis, E.; Luisi, B. and Hughes, C (2000) Nature, 405, 914-9.

[47] Akama, H.; Matsuura, T.; Kashiwagi, S.; Yoneyama, H.; Narita, S.; Tsukihara, T.; Nakagawa, A. and Nakae, T. (2004) J. Biol. Chem., 279, 25939-42

[48] Federici, L.; Du, D.; Walas, F.; Matsumura, H.; Fernandez-Recio, J.; McKeegan, K. S.; Borges-Walmsley, M. I.; Luisi, B. F, and Walmsley, A. R. (2005) J. Biol. Chem., 280, 15307-14.

[49] Andersen, C.; Koronakis, E.; Bokma, E.; Eswaran, J.; Humphreys, D.; Hughes, C. and Koronakis, V. (2002) Proc. Natl. Acad. Sci. USA, 99, 11103-8

[50] Eswaran, J.; Hughes, C. and Koronakis, V. (2003) J. Mol. Biol, 327, 309-15.

[51] Augustus, A. M.; Celaya, T.; Husain, F.; Humbard, M. and Misra, R. (2004) J. Bacteriol., 186, 1851-60.
[52] Dinh, T.; Paulsen, I. T.; Saier, M. H., Jr. (1994) J. Bacteriol., 176 3825-31

[53] Tikhonova, E. B.; Devroy, V. K.; Lau, S. Y. and Zgurskaya, H. I. (2007) Mol. Microbiol., 63, 895-910.

[54] Higgins, M. K.; Bokma, E.; Koronakis, E.; Hughes, C and Koronakis, V. (2004) Proc. Natl. Acad. Sc.i U. S. A., 101, 9994-9.

[55] Mikolosko, J; Bobyk, K.; Zgurskaya, H. I. and Ghosh, P. (2006) Structure, 14, 577-87.

[56] Touze, T.; Eswaran, J.; Bokma, E.; Koronakis, E.; Hughes, C. and Koronakis, V. (2004) Mol. Microbiol., 53, 697-706.

[57] Zgurskaya, H. I. and Nikaido, H. (1999) J. Mol. Biol., 285, 409-20

[58] Yoneyama, H.; Maseda, H.; Kamiguchi, H. and Nakae, T. (2000) J. Biol. Chem., 275, 4628-34

[59] Zgurskaya, H. I. and Nikaido, H. (2000) J. Bacteriol., 182, 4264-7.

[60] Stenberg, F.; Chovanec, P.; Maslen, S. L.; Robinson, C. V.; Ilag, L. L.; von Heijne, G. and Daley, D. O. (2005) J. Biol. Chem., 280, 34409-19.

[61] Murakami, S.; Nakashima, R.; Yamashita, E. and Yamaguchi, A. (2002) Nature, 419, 587-93.

[62] Pos, K. M. and Diederichs, K. (2002) Acta. Crystallogr. Biol. Crystallogr, D58, 1865-7.

[63] Pos, K. M.; Schiefner, A.; Seeger, M. A. and Diederichs, K. (2004) FEBS Lett., 564, 333-9.

[64] Eda, S.; Yoneyama, H. and Nakae, T. (2003) Biochemisyry, 42 7238-44

[65] Guan, L. and Nakae, T. (2001) J. Bacteriol., 183, 1734-9.

[66] Takatsuka, Y. and Nikaido, H. (2006) J. Bacteriol., 188, 7284-9.

[67] Seeger, M. A.; Schiefner, A.; Eicher, T.; Verrey, F.; Diederichs, K. and Pos, K. M. (2006) Science, 313, 1295-8

[68] Murakami, S.; Nakashima, R.; Yamashita, E.; Matsumoto, T. and Yamaguchi, A. (2006) Nature, 443, 173-9.

[69] Sennhauser, G.; Amstutz, P.; Briand, C.; Storchenegger, O. and Grutter, M. G. (2007) PLoS Biol., 5, e7.

[70] Yu, E. W.; McDermott, G.; Zgurskaya, H. I.; Nikaido, H. and Koshland, D. E., Jr. (2003) Science, 300, 976-80.

[71] Yu, E. W.; Aires, J. R.; McDermott, G. and Nikaido, H. (2005) J Bacteriol., 187, 6804-15.

[72] Tornroth-Horsefield, S.; Gourdon, P.; Horsefield, R.; Brive, L.; Yamamoto, N.; Mori, H.; Snijder, A. and Neutze, R. (2007) Structure, 15, 1663-73.

[73] Elkins, C. A. and Nikaido, H. (2002) J. Bacteriol., 184, 6490-8.

[74] Eda, S.; Maseda, H. and Nakae, T. (2003) J. Biol. Chem., 278, 2085-8

[75] Tikhonova, E. B.; Wang, Q. and Zgurskaya, H. I. (2002) J. Bacteriol., 184, 6499-507

[76] Mao, W: Warren, M. S.; Black, D. S.; Satou, T; Murata, T.; Nishino, T:; Gotoh, N. and Lomovskaya, O. (2002) Mol. Microbiol., 46, 889-901.

[77] Murakami, S.; Tamura, N.; Saito, A.; Hirata, T. and Yamaguchi, A. (2004) J. Biol. Chem., 279, 3743-8.

[78] Seeger, M. A.; von Ballmoos, C; Eicher, T.; Brandstatter, L.; Verrey, F.; Diederichs, K. and Pos, K. M. (2008) Nat. Struct. Mol. Biol., 15, 199-205

[79] Zgurskaya, H. I. and Nikaido, H. (1999) Proc. Natl. Acad. Sci. USA, 96, 7190-5.

[80] Aires, J. R. and Nikaido, H. (2005) J. Bacteriol., 187, 1923-9

[81] Tikhonova, E. B. and Zgurskaya, H. I. (2004) J. Biol. Chem., 279 32116-24.

[82] Nehme, D.; Li, X. Z.; Elliot, R. and Poole, K. (2004) J. Bacteriol., 186, 2973-83

[83] Tamura, N.; Murakami, S.; Oyama, Y.; Ishiguro, M. and amaguchi, A. (2005) Biochemistry, 44, 11115-21.

[84] Lobedanz, S.; Bokma, E.; Symmons, M. F.; Koronakis, E.; Hughes, C. and Koronakis, V. (2007) Proc. Natl. Acad. Sci. USA, 104, $4612-7$

[85] Mokhonov, V. V.; Mokhonova, E. I.; Akama, H. and Nakae, T. (2004) Biochem. Biophys. Res. Commun., 322, 483-9.

[86] Gerken, H.; Misra, R. (2004) Mol. Microbiol., 54, 620-31.

[87] Eda, S.; Maseda, H; Yoshihara, E. and Nakae, T. (2006) FEMS Microbiol. Lett., 254, 101-7.

[88] Stegmeier, J. F.; Polleichtner, G.; Brandes, N.; Hotz, C. and Andersen, C. (2006) Biochemistry, 45, 10303-12.

[89] Bokma, E.; Koronakis, E.; Lobedanz, S.; Hughes, C. and Koronakis, V. (2006) FEBS Lett., 580, 5339-43.

[90] Vediyappan, G.; Borisova, T. and Fralick, J. A. (2006) J. Bacteriol. $188,3757-62$ 
[91] Nehme, D.; Poole, K. (2005) Antimicrob. Agents Chemother, 49, 4375-8.

[92] Krishnamoorthy, G.; Tikhonova, E. B. and Zgurskaya, H. I. (2008) J. Bacteriol., 190, 691-8

[93] Vaccaro, L.; Koronakis, V. and Sansom, M. S. (2006) Biophys. J., 91, 558-64.

[94] Su, C. C.; Li, M.; Gu, R.; Takatsuka, Y.; McDermott, G.; Nikaido, H. and Yu, E. W. (2006) J. Bacteriol., 188, 7290-6

[95] Das, D.; Xu, Q. S.; Lee, J. Y.; Ankoudinova, I.; Huang, C.; Lou Y.; DeGiovanni, A.; Kim, R. and Kim, S. H. (2007) J. Struct. Biol., 158, 494-502.

[96] Abrahams, J. P.; Leslie, A. G.; Lutter , R. and Walker, J. E. (1994) Nature, 370, 621-8

[97] Hackney, D. D.; Rosen, G. and Boyer, P. D. (1979) Proc. Natl. Acad. Sci. USA, 76, 3646-50.
[98] Hutton, R. L. and Boyer, P. D. (1979) J. Biol. Chem., 254, 9990-3.

[99] Boyer, P. D. (1997) Annu. Rev. Biochem., 66, 717-49.

[100] Petrek M., O. M., Banas P., Koca J. and Damborsky J. (2006)

[101] Petrek, M.; Kosinova, P.; Koca, J. and Otyepka, M. (2007) Structure, 15, 1357-63.

[102] Su, C. C.; Nikaido, H. and Yu, E. W. (2007) FEBS Lett., 581, 4972-6

[103] Lewinson, O. and Bibi, E. (2001) Biochemistry, 40, 12612-8.

[104] Muth, T. R. and Schuldiner, S. (2000) EMBO J., 19, 234-40.

[105] Lewinson, O.; Adler, J.; Poelarends, G. J.; Mazurkiewicz, P.; Driessen, A. J. and Bibi, E. (2003) Proc. Natl. Acad. Sci. USA, 100, 1667-72.

[106] Li, X. Z.; Ma, D.; Livermore, D. M. and Nikaido, H. (1994) Antimicrob. Agents Chemother., 38, 1742-52. 